\title{
A WRKY transcription factor, TaWRKY42-B, facilitates initiation of leaf senescence by promoting jasmonic acid biosynthesis
}

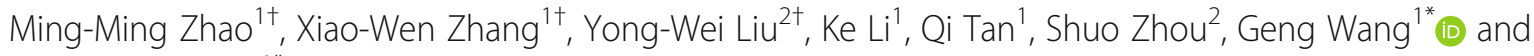
Chun-Jiang Zhou ${ }^{1 *}$

\begin{abstract}
Background: Leaf senescence comprises numerous cooperative events, integrates environmental signals with agedependent developmental cues, and coordinates the multifaceted deterioration and source-to-sink allocation of nutrients. In crops, leaf senescence has long been regarded as an essential developmental stage for productivity and quality, whereas functional characterization of candidate genes involved in the regulation of leaf senescence has, thus far, been limited in wheat.

Results: In this study, we analyzed the expression profiles of 97 WRKY transcription factors (TFs) throughout the progression of leaf senescence in wheat and subsequently isolated a potential regulator of leaf senescence, TaWRKY42-B, for further functional investigation. By phenotypic and physiological analyses in TaWRKY42-Boverexpressing Arabidopsis plants and TaWRKY42-B-silenced wheat plants, we confirmed the positive role of TaWRKY42-B in the initiation of developmental and dark-induced leaf senescence. Furthermore, our results revealed that TaWRKY42-B promotes leaf senescence mainly by interacting with a JA biosynthesis gene, AtLOX3, and its ortholog, TaLOX3, which consequently contributes to the accumulation of JA content. In the present study, we also demonstrated that TaWRKY42-B was functionally conserved with AtWRKY53 in the initiation of age-dependent leaf senescence.

Conclusion: Our results revealed a novel positive regulator of leaf senescence, TaWRKY42-B, which mediates JArelated leaf senescence via activation of JA biosynthesis and has the potential to be a target gene for molecular breeding in wheat.
\end{abstract}

Keywords: Leaf senescence, WRKYs, JA, Transcriptional regulation, Wheat

\footnotetext{
* Correspondence: gengwang@hebtu.edu.cn; cjzhou@hebtu.edu.cn

${ }^{\dagger}$ Ming-Ming Zhao, Xiao-Wen Zhang and Yong-Wei Liu contributed equally to this work.

${ }^{1}$ Ministry of Education Key Laboratory of Molecular and Cell Biology, Hebei Collaboration Innovation Center for Cell Signaling, College of Life Sciences, Hebei Normal University, Shijiazhuang 050024, Hebei, China

Full list of author information is available at the end of the article
}

(c) The Author(s). 2020 Open Access This article is licensed under a Creative Commons Attribution 4.0 International License, which permits use, sharing, adaptation, distribution and reproduction in any medium or format, as long as you give appropriate credit to the original author(s) and the source, provide a link to the Creative Commons licence, and indicate if changes were made. The images or other third party material in this article are included in the article's Creative Commons licence, unless indicated otherwise in a credit line to the material. If material is not included in the article's Creative Commons licence and your intended use is not permitted by statutory regulation or exceeds the permitted use, you will need to obtain permission directly from the copyright holder. To view a copy of this licence, visit http://creativecommons.org/licenses/by/4.0/. The Creative Commons Public Domain Dedication waiver (http://creativecommons.org/publicdomain/zero/1.0/) applies to the data made available in this article, unless otherwise stated in a credit line to the data. 


\section{Background}

Leaf senescence is a highly integrated developmental stage that coordinates multidimensional alterations at the physiological and molecular level [1]. During leaf senescence, intracellular organelles and macro-molecules are dismantled and degenerated, which predominantly contributes to the source-to-sink allocation of nutrients [2]. The redistribution of nutrients from senescent organs to vegetative tissues is essential for plant fitness and critical for productivity and quality in crops [3, 4]. Generally, the initiation of leaf senescence is age-dependent and triggered by developmental cues [5]. Additionally, leaf senescence is tightly controlled by epigenetic modification, circadian clock, autophagy, chlorophyll metabolism, and transcriptional regulation [6-17]. Meanwhile, when plants encounter abiotic and biotic stresses, the initiation of senescence will be accelerated $[5,18]$.

Phytohormones play both positive and negative roles in the onset of leaf senescence. Among the leaf senescence-related hormones, ethylene, abscisic acid (ABA), jasmonic acid (JA), salicylic acid (SA), brassinosteroids (BRs), and strigolactones (SLs) facilitate leaf senescence initiation, while cytokinins (CKs), gibberellic acid (GA), and auxins (AUXs) retard leaf senescence [19-28].

JA, a kind of lipid derived phytohormone, is vital for biotic stress defense, abiotic stress tolerance, and developmental regulation in plants $[29,30]$. The functional role of JA in promoting leaf senescence was first identified by the severe degradation of chlorophyll in oat (Avena sativa L. cv "Victory") leaves after application of methyl jasmonate (MeJA) in 1980 [31]. Meanwhile, the exogenous application of JA to wild-type Arabidopsis can elevate the transcripts of senescence-related genes, such as SAG12 and SEN4, but not coi1-1. Moreover, expression of many JA biosynthesis genes and JA content are enhanced in senescent leaves [32]. A lipoxygenase for JA production, AtLOX2, is under the regulation of TCP4 in JA-induced leaf senescence, which is simultaneously repressed by miRNA319 [33, 34]. Recently, studies on key components of the JA signaling pathway manifested that Arabidopsis MYC2, MYC3, and MYC4 promote leaf senescence by directly binding to the promoter of SAG29; this interaction is antagonistically regulated by III d group bHLHs [21]. However, the process of leaf senescence in coil-1 and some JA-related mutants is similar to the wild type (WT), suggesting that the dissection of the intricate mechanism underlying JA-induced leaf senescence is of immense importance [32, 35].

In addition to hormone responsive genes, numerous senescence-related genes, such as senescence associated genes (SAGs), have been proven to be crucial for the timing of leaf senescence [36]. Among which, senescencerelated transcription factors, including NACs, WRKYs,
bZIPs, MYCs, and MYBs, integrate various senescence signals into the nucleus and consequently execute transcriptional modulation $[15,16,37]$. WRKYs are named after the conserved WRKY domain that possesses a WRKYGQK core motif and a zinc-finger motif and is one of the biggest TF families in plants. Based on structural features and phylogenetic analysis, WRKYs are further divided into seven subgroups (I, IIa + IIb, IIc, IId + IIe, and III). To date, WRKYs are reported to participate in multiple stress-defense responses and developmental regulations. In addition, WRKYs involved in the modulation of leaf senescence have been widely acknowledged $[38,39]$. Among those senescence-related WRKYs, AtWRKY53 has been intensively studied for its critical role in promoting leaf senescence initiation [40-42]. AtWRKY6 plays a role in GA- and light-related senescence processes [43-45]. AtWRKY57 coordinates JA and Aux signaling for fine tuning leaf senescence [46]. AtWRKY54 and AtWRKY70, two functionally redundant WRKYs, function as negative regulators in leaf senescence [47]. AtWRKY45 and AtWRKY75 trigger the initiation of leaf senescence via the GA and SA signaling pathway, respectively $[22,26]$. Collectively, WRKYs are competent to integrate diverse signals for promoting and retarding leaf senescence onset in Arabidopsis. However, how WRKYs regulate leaf senescence in crops is still largely unknown.

Bread wheat (Triticum aestivum L.) is one of the most widely cultivated crops and serves as a staple food resource for more than $30 \%$ of the world's population, providing approximately $20 \%$ of the calories consumed by humans [48]. The allohexaploid genome of bread wheat (AABBDD) arises from two polyploidization events. The first hybridization between diploid progenitors Triticum urartu $(2 \mathrm{n}=2 \mathrm{x}=14$, genome $\mathrm{AA})$ and the section Sitopsis (donor of genome BB, presumably related to Aegilops speltoides, $2 \mathrm{n}=2 \mathrm{x}=14$, genome SS) resulted in the formation of the allotetraploid ancestor Triticum turgidum $(2 \mathrm{n}=4 \mathrm{x}=28$; genome $\mathrm{AABB})$. The second hybridization between Triticum turgidum and Aegilops tauschii $(2 \mathrm{n}=2 \mathrm{x}=14$, genome $\mathrm{DD})$ finally contributed to the origination of bread wheat $[49,50]$. Due to the allohexaploid nature of bread wheat, the sequencing of the genome and the characterization of functional genes have lagged behind those of other crops. Fortunately, databases established by next-generation sequencing technologies and whole-genome shotgun sequencing provide new approaches for wheat genome study [51-54]. However, candidate genes in the regulation of leaf senescence remain elusive and need to be explored to improve productivity and grain quality in wheat.

In this study, we aimed to screen the candidate genes of leaf senescence by an RNA-sequencing (RNA-seq) 
experiment in wheat. Consequently, we selected a WRKY type TF, TaWRKY42-B, for further functional research. Intriguingly, despite the expression levels of TaWRKY42- $B$, it was negatively correlated with the progression of leaf senescence, the silencing of TaWRKY42$B$ drastically delayed the leaf senescence process in wheat, and the overexpression of TaWRKY42-B in Arabidopsis caused premature leaf senescence, implying that TaWRKY42-B functioned as a positive regulator of leaf senescence. Subsequently, we demonstrated that TaWRKY42-B modulated leaf senescence was related to JA signaling through physiological and molecular analysis. We further manifested that TaWRKY42-B can promote JA biosynthesis by interacting with a JA biosynthesis gene AtLOX3 and its ortholog (TaLOX3, TraesCS4B02G295200), altering the transcription of AtLOX3 and TaLOX3. Moreover, the TaWRKY42-BTaLOX3 module underwent continuous suppression after leaf senescence onset, which possibly contributed to the organized progression of leaf senescence. Through complementary assay, we also showed that TaWRKY42$B$ had a partially conserved function with AtWRKY53 in the regulation of age-dependent leaf senescence. Briefly, our findings revealed the functional role of a new senescence-related TF, TaWRKY42-B, which is a positive and JA-related regulator of leaf senescence in wheat. Our data also suggest that TaWRKY42-B potentially serves as a target gene for molecular breeding in wheat.

\section{Results}

\section{TaWRKY42-B is downregulated during leaf senescence}

To identify candidate genes in the regulation of leaf senescence in T. aestivum (cv Chinese Spring), we conducted an RNA-seq experiment at four developmental stages of flag leaves (YL, young leaves; ML, mature leaves; ES, early senescent leaves; LS, late senescent leaves). For each sample, three biological replicates were employed. At senescent stages, we identified 32 up-regulated WRKYs and 65 down-regulated WRKYs compared with young leaf stage ( $\mid \log 2$ (fold change) $\mid \geq 1 ; P<0.05$ ) (Fig. 1a-b). Among the senescence-related TaWRKYs in this study, TaWRKY42-B (TraesCS2B02G187500), a member of the group III WRKYs (Additional file 1: Fig. S1a), exhibited the maximum expression level at ML stage and the decreasing expression through the progression of leaf senescence in flag leaves (Fig. 1c). To verify the expression pattern of TaWRKY42- $B$, we inspected the spatial-temporal transcriptional profiling of TaWRKY42-B in wheat. First, the negative correlation between transcription of TaWRKY42$B$ and age-dependent senescence process in wheat flag leaves was confirmed by quantitative real time-PCR (qRTPCR) assay (Fig. 2d). Leaf senescence-associated parameters including chlorophyll content and ion leakage rate (Fig. 2b, c), as well as the transcription level of two SAGs,
TaSAG3 and TaSAG5 (Fig. 2e, f), were examined for the propriety of harvesting flag leaves at distinctive senescence stages (Fig. 2a). Generally, senescence proceeded from the leaf tip and gradually progressed toward the leaf base [55] (Fig. 2g). Consistently, fewer transcripts of TaWRKY42-B were detected in the leaf tip than the middle and base (Fig. 2h), which was opposite to that of TaSAG3 (Fig. 2i). Next, the transcription level of TaWRKY42-B was analyzed among different tissues, including spike, seed, root, stem, flag leaf, and mature leaf (Fig. 3a). TaWRKY42-B exhibited a ubiquitous expression pattern in wheat, whereas the most abundant transcripts of TaWRKY42-B were detected in flag leaves (Fig. $3 \mathrm{~b}$ ). The above data suggested that TaWRKY42-B is one of the senescence downregulated genes (SDGs) and may predominantly function in leaves.

TaWRKY42-B plays a positive role in age-dependent and dark-induced leaf senescence

To further functionally characterize TaWRKY42-B, TaWRKY42-B was silenced by the Barley stripe mosaic virus (BSMV)-VIGS method in wheat with a 198 bp target sequence specifically selected from $679 \mathrm{bp}$ downstream of the translation start codon of the TaWRKY42$B$ coding sequence (CDS) but not in TaWRKY42- $A$ and TaWRKY42-D (Additional file 1: Fig. S1b, c and Additional file 2: Fig. S2a), and the empty vector (pCaBS$\gamma$ bLIC) was used as a control. Seven-day-old wheat seedlings were infected with BSMV that harbored BSMV:: TaWRKY42- ${ }_{198}$ (pCaBS- $\alpha$, pCaBS- $\beta$, and pCaBSүbTaWRKY42-B 198 ) (Additional file 2: Fig. S2b). After growth for another 25 days, the 8 th leaf from the top of the vector control (VC) and TaWRKY42-B-silenced plants were detached and incubated in the dark for 6 days. TaWRKY42-B-silenced leaves exhibited an obviously delayed leaf senescence phenotype compared to VC transgenic leaves (Fig. 4a). Consistently, chlorophyll degradation, ion leakage rate, and expression of TaSAG3 and TaSAG5 in TaWRKY42-B-silenced leaves were remarkably decreased compared with those of $\mathrm{VC}$ plants (Fig. 4f-i). Accumulation of hydrogen peroxide $\left(\mathrm{H}_{2} \mathrm{O}_{2}\right)$ in senescent leaves has long been known for the subsequent oxidative damage. Less dark brown spots existed on TaWRKY42-B-silenced leaves after 3,3-diaminobenzidine (DAB) staining, which demonstrated that the senescence process was delayed by silencing of TaWRKY42-B (Fig. 4c). To estimate whether the delayed leaf senescence resulted from the depressed expression level of TaWRKY42-B, the transcription level of TaWRKY42-A, TaWRKY42-B, and TaWRKY42-D was examined in those infected wheat plants. Notably, the stay-green phenotype was clearly observed after dark treatment in detached leaves with diminished transcription of TaWRKY42-B (Fig. 4b) and comparable levels of 
a

b
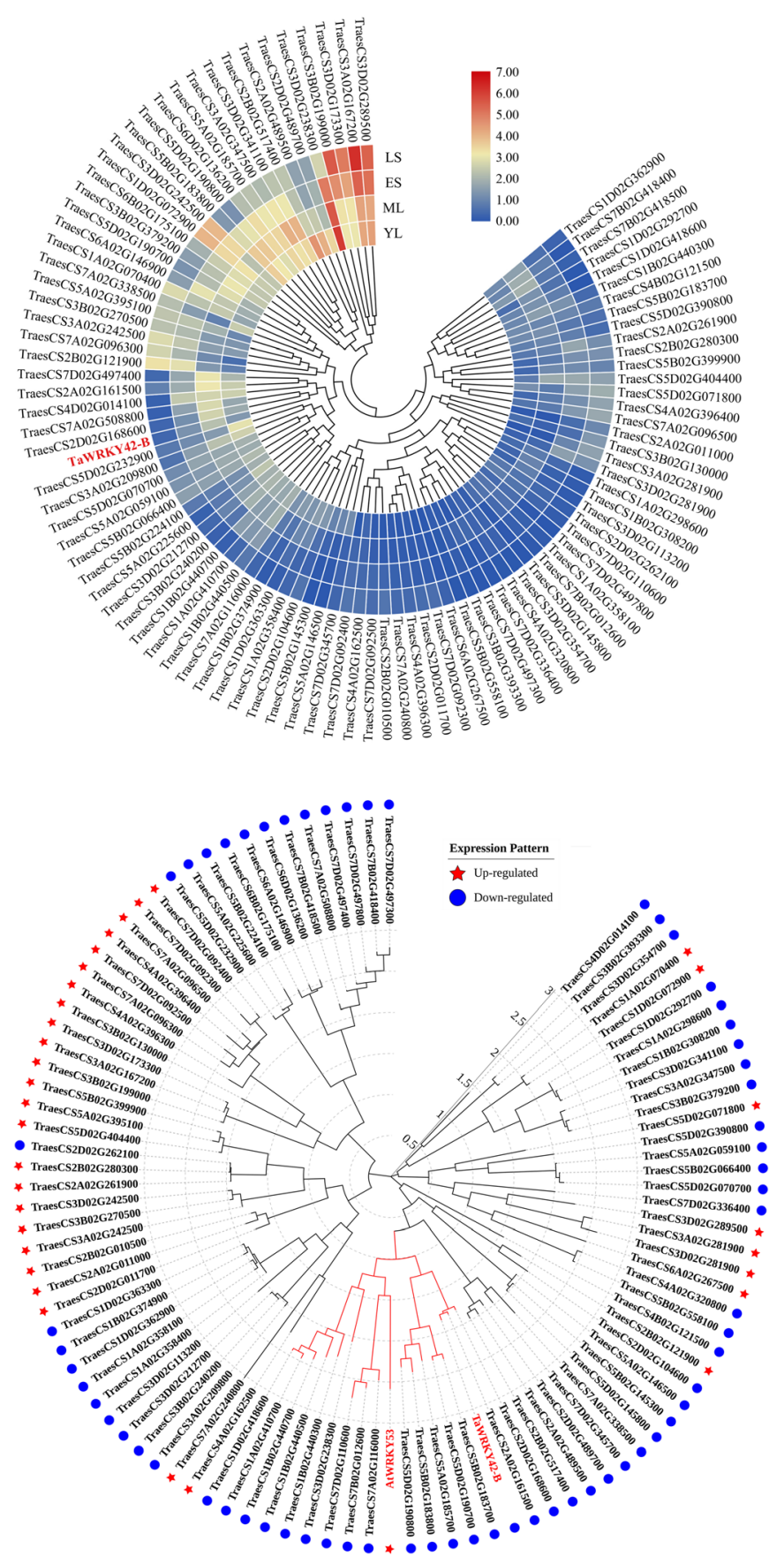

c

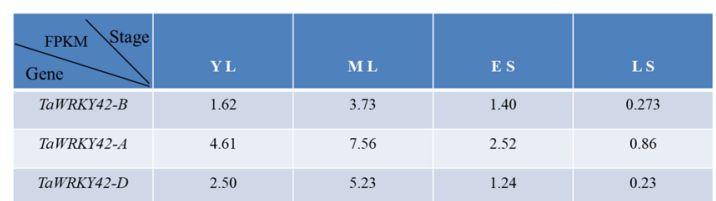

Fig. 1 (See legend on next page.) 
(See figure on previous page.)

Fig. 1 Expression profiling of TaWRKYs at different senescence stages. a Heatmap depiction of TaWRKYs among four developmental leaf stages. Blue indicates the expression level of TaWRKYs decreased relative to $Y L$, whereas red indicates the transcription level of TaWRKYS relative to $Y L$. ( $Y L$, young leaves; ML, mature leaves; ES, early senescent leaves, with $<25 \%$ leaf area yellowing; LS, late senescent leaves with $>50 \%$ leaf area yellowing). b Phylogenetic analysis of senescence-related TaWRKYs. c Transcription level (FPKM value) of TaWRKY42-B, TaWRKY42-A and TaWRKY42$D$ were monitored through the progression of leaf senescence. (Dots and asterisks indicate down and up-regulated genes)

$\mathrm{a}$

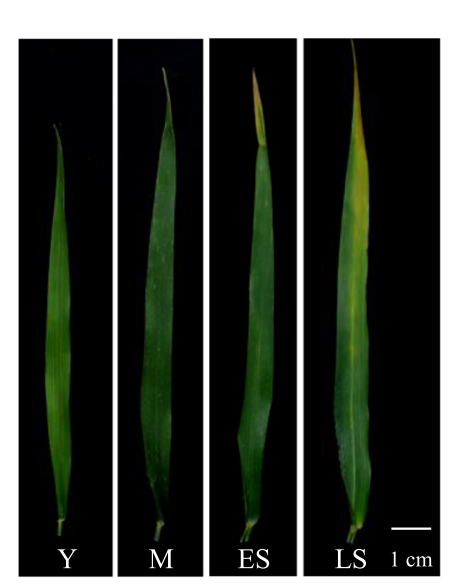

d

TaWRKY42-B

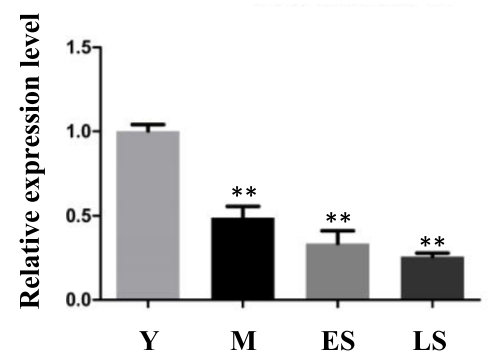

g

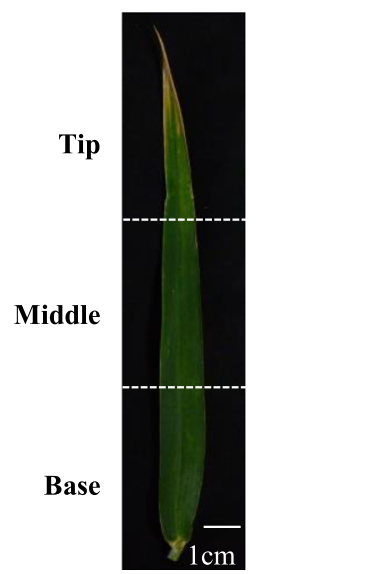

b

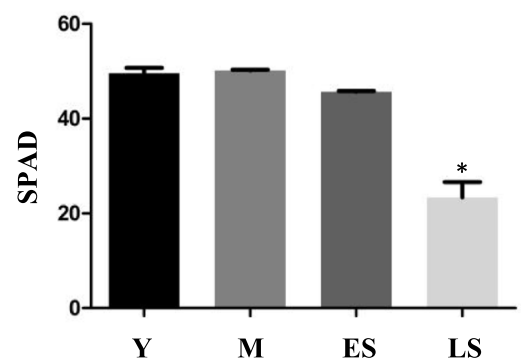

e

TaSAG3

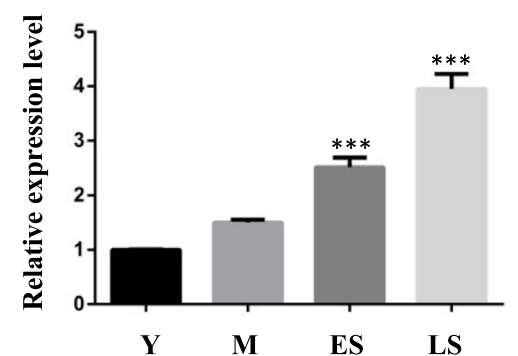

$\mathrm{h}$

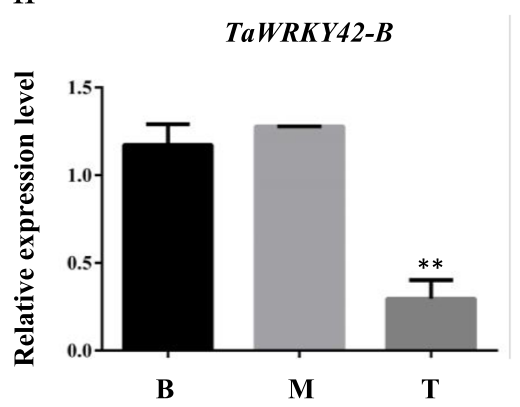

\section{c}

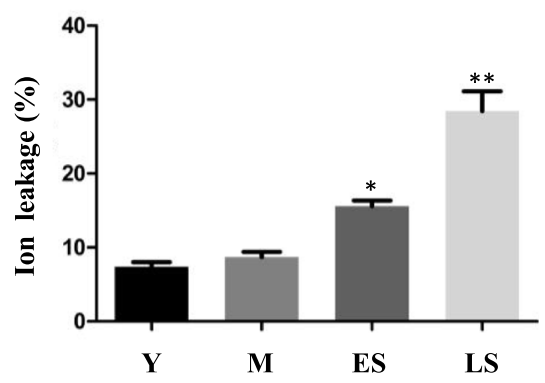

f

TaSAG5

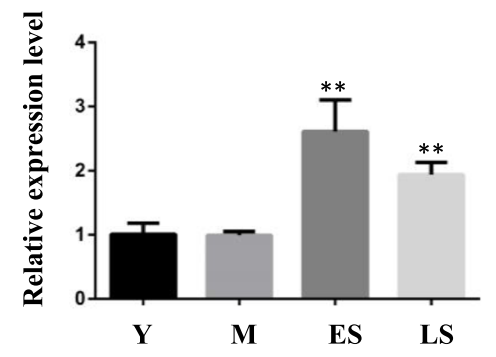

i

TaSAG3

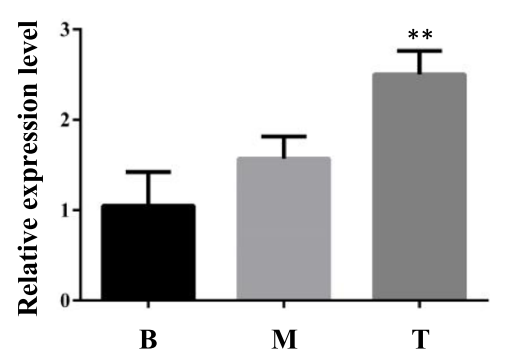

Fig. 2 Spatiotemporal expression pattern of TaWRKY42-B in wheat. a Expression pattern of TaWRKY 42-B was detected at four different developmental stages of wheat flag leaves (Y, M, ES, and LS). Chlorophyll content (b) and ion leakage rate (c) of leaves as shown in (a). d-f Transcription level of senescence-associated genes TaWRKY42-B, TaSAG3 and TaSAG5 in leaves as shown in (a). g-i Expression level of TaWRKY42-B and TaSAG3 in tip, middle and base of a senescent wheat flag leaf. (Error bars indicate standard deviation (SD). Asterisks indicate significant differences. Student's t-test, ${ }^{*} P<0.05,{ }^{* *} P<0.01,{ }^{* * *} P<0.001$ ) 
a

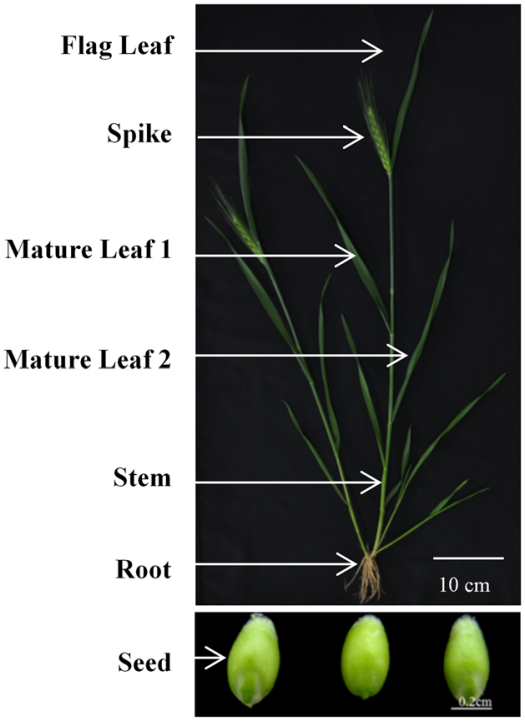

c

TaWRKY 42-B-GFP

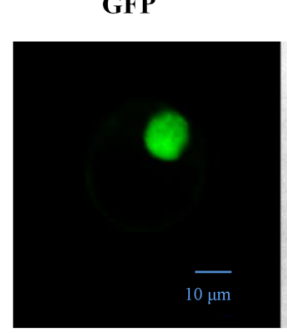

GFP

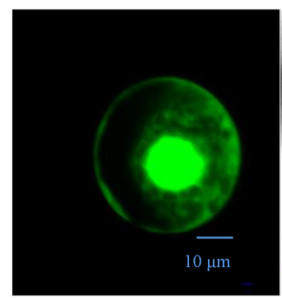

d

Reporter:

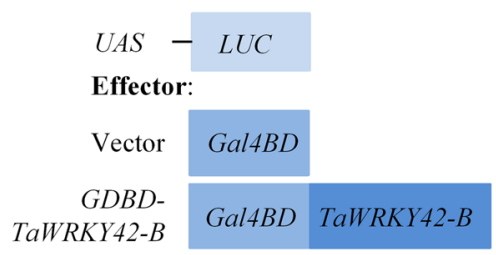

Internal control :

$35 S-R E N$ b

TaWRKY42-B
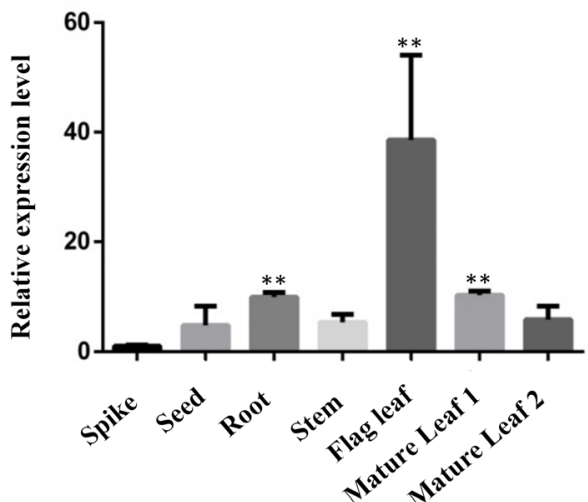

Bright

Merged
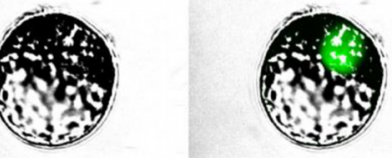

$10 \mu \mathrm{m}$
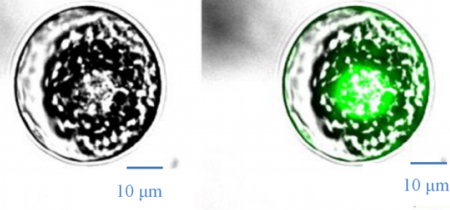

e

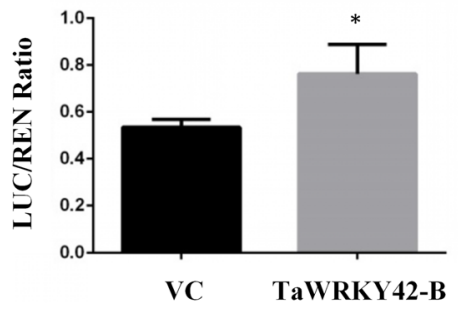

Fig. 3 (See legend on next page.) 
(See figure on previous page.)

Fig. 3 Tissue and subcellular expression pattern of TaWRKY42-B in wheat. a-b Tissue expression pattern of TaWRKY42-B was examined in different tissues (Flag leaf, Spike, 1st mature leaf from the top, Mature leaf 1; 2nd mature leaf from the top, Mature leaf 2; Stem, Root, and Seed) of 6month-old wheat plants by qRT-PCR. c Subcellular localization of TaWRKY42-B-GFP fusion protein in the wheat protoplast. The control experiment employed a simple GFP protein. Bars $=10 \mu \mathrm{m}$. All experiments were repeated at least three times. $\mathbf{d}$ Diagrams of constructs in the transcriptional activity assay. e Measurement of relative LUC reporter activity after transient expression of vectors as shown in (d). (Error bars indicate SD. Asterisks indicate significant differences. Student's t-test, ${ }^{*} P<0.05,{ }^{*} P<0.01$ )

TaWRKY42-A and TaWRKY42-D to that of the control leaves (Fig. 4d, e). This result suggested that TaWRKY42-B was a positive regulator of leaf senescence onset in wheat.

To further assess the functional role of TaWRKY42-B in leaf senescence, TaWRKY42-B-overexpressing (OE) Arabidopsis plants were generated. A full-length $879 \mathrm{bp}$ CDS of TaWRKY42-B was cloned into pCAMBIA1300 and fused with green fluorescent protein (GFP) tag, which was driven by the Cauliflower mosaic virus (CaMV) 35S promoter. Two independent homozygous transgenic lines $(O E-1$ and $O E-2)$ were selected for further phenotypic and physiological analysis. The elevated expression level of TaWRKY42-B was confirmed by a western blot in $O E-1$ and $O E-2$ (Fig. 5b). Next, we observed that 5-week-old $O E-1$ and $O E-2$ plants showed a precocious leaf senescence phenotype compared with Col-0 (Fig. 5a, c). The chlorophyll content of TaWRKY42-B-OE lines decreased in Col-0 (Fig. 5d) and ion leakage rates were accelerated by overexpression of TaWRKY42-B (Fig. 5f). More $\mathrm{H}_{2} \mathrm{O}_{2}$ was detected by DAB staining in TaWRKY42-B-OE lines (Fig. 5e). Consistent with the observed phenotypic alterations, two SAGs, AtSAG12 and AtSEN4, were upregulated in $O E-1$ and $O E-2$ (Fig. $5 \mathrm{~g}, \mathrm{~h}$ ), while two SDGs, AtRBCS and $A t C A B 1$, were suppressed in TaWRKY42-B-OE (Fig. 5i, j). To investigate whether TaWRKY42-B was involved in dark-induced leaf senescence in Arabidopsis, we detached the 8th rosette leaves from $O E-1, O E-2$, and Col-0, which were apparently undistinguishable and subsequently, incubated under darkness for another 6 days (Additional file 5: Fig. S5a). Senescence-related phenotypic alterations, chlorophyll degradation and ion leakage rate were more dramatically changed in $O E-1$ and $O E-2$ after dark treatment (Additional file 5: Fig. S5b, c). The above data validated that TaWRKY42-B promoted leaf senescence under both optimal growth conditions and darkness.

\section{TaWRKY42-B localizes in the nucleus and possesses transcriptional activity}

Generally, WRKYs are implicated in transcriptional regulation of diverse biological processes. To illustrate whether TaWRKY42-B plays a role in transcriptional modulation, we produced a 35S:TaWRKY42-B-GFP construct, which was then transformed and transiently expressed into wheat protoplasts. GFP fluorescence signals of TaWRKY42-B-GFP fusion predominantly concentrated in the nucleus, while signals of GFP alone were visualized in the plasma membrane, cytoplasm, and nucleus (Fig. 3c). This specific subcellular localization of TaWRKY42-B implied that TaWRKY42-B potentially participated in transcriptional regulation in wheat. TaWRKY-42-B showed strong transcriptional activity in yeast (Additional file 3: Fig. S3). Consequently, the transcriptional activity of TaWRKY42-B in wheat protoplasts was detected by a dual-luciferase reporter system. TaWRKY42-B was fused to a Gal4 DNA-binding domain (GDBD) and co-transfected with the firefly luciferase (LUC) gene, which was under the control of an upstream activating sequence (UAS) and the Renilla luciferase (REN) gene, driven by a CaMV 35S promoter fusion was used as an internal control (Fig. 3d). After the addition of enzymatic substrates, GDBD-TaWRKY42-B, but not GDBD only, was able to facilitate the enzymatic reaction of LUC (Fig. 3e). These results indicated that TaWRKY42-B was a nucleus-localized TF.

\section{JA is essential for TaWRKY42-B to trigger leaf senescence}

To uncover the mechanism underlying TaWRKY42-Binduced leaf senescence, we searched cis-acting elements that were distributed in the TaWRKY42-B promoter region for clues. Noticeably, three CGTCA-motifs related to JA responsiveness lay in the TaWRKY42-B promoter region $(-980,-1865$, and $-2009 \mathrm{bp}$ ) (Additional file 4 : Fig. S4). In addition, after the application of $200 \mu \mathrm{M}$ MeJA, transcription levels of TaWRKY42-B were at a maximum after $2 \mathrm{~h}$ and then decreased to a relatively low level from 4 to $24 \mathrm{~h}$ (Fig. 6c). For decades, JA has been well acknowledged as a leaf senescence-related phytohormone. Therefore, we speculated that TaWRKY42-B modulated leaf senescence in a JA-related manner. Rosette leaves were harvested from Col-0, $O E$ 1 , and $O E-2$ and treated with $100 \mu \mathrm{M}$ MeJA for 4 days. JA-induced leaf senescence appeared in both Col-0 and TaWRKY42-B-OE plants, but lower chlorophyll content and more severe ion leakage were found only in $O E-1$ and $O E-2$ (Fig. 6a, b, and d). To finely dissect the details of the interaction between TaWRKY42-B and the JA signaling pathway, the expression level of a series of JArelated genes were inspected. JA biosynthesis genes, including AtLOX1, AtLOX2, AtLOX3, and a JA-responsive gene, AtVSP2, were elevated in $O E-1$ and $O E-2$ when 


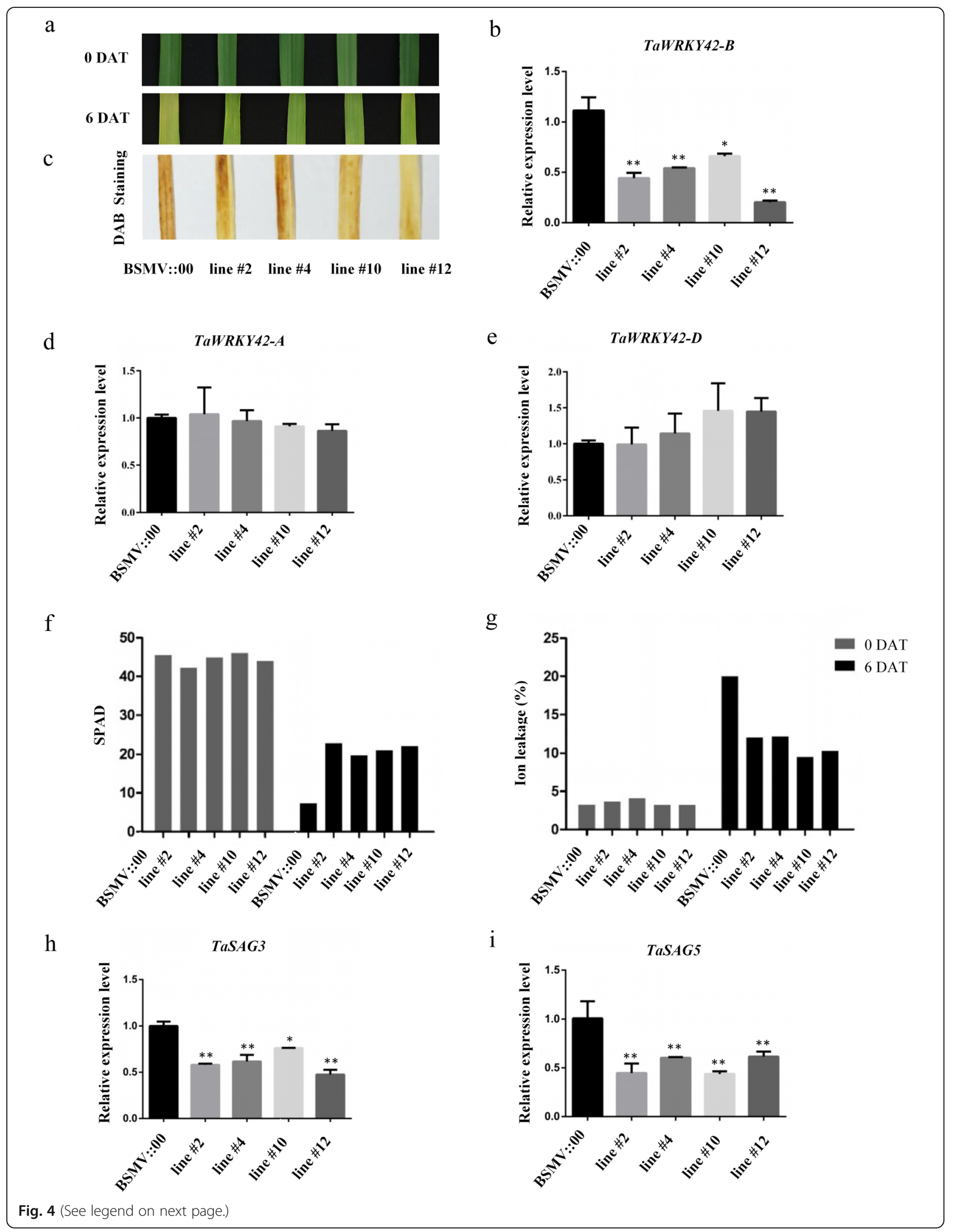


(See figure on previous page.)

Fig. 4 Silencing of TaWRKY42-B delays leaf senescence in wheat. a Phenotypic analysis of detached leaves of TaWRKY42-B-silenced plants and controls under darkness for 6 days. (DAT, Day after treatment). b Transcription level of TaWRKY42-B in leaves of TaWRKY42-B-silenced plants and controls. c DAB staining of detached leaves after dark treatment as shown in (a). d-e The expression level of TaWRKY42-B orthologs (TaWRKY42-A and TaWRKY42-D) in detached leaves of TaWRKY42-B-silenced plants and controls. Chlorophyll content (f) and ion leakage rate (g) of detached leaves as shown in (a). $\mathbf{h}-\mathbf{i}$ Transcription level of senescence-associated genes TaSAG3, and TaSAG5 of detached leaves as shown in (a). (Error bars indicate SD. Asterisks indicate significant differences. Student's $t$-test, ${ }^{*} P<0.05,{ }^{*} P<0.01$ )

compared to Col-0 (Fig. 7a-d). However, signaling component genes, such as AtMYC2 and AtMYC3, were nearly unaffected by overexpression of TaWRKY42-B (Fig. 7e-f). Meanwhile, the expression of AtMYC4 was slightly decreased in TaWRKY42-B-OE plants (Fig. 7g). These results suggested that TaWRKY42-B promoted leaf senescence mainly by altering the expression of JA biosynthesis genes.

TaWRKY42-B directly binds to the promoter region of LOXs WRKYs have long been known to regulate the transcription of diverse target genes, which is generally accomplished by the interaction between WRKYs and W-box contained promoter regions. We identified one and two W-box motifs in AtLOX1 and AtLOX3 promoters, respectively (Additional file 6: Fig. S6a and Fig. 8a). Thus, we investigated whether TaWRKY42-B binds to the Wboxes in AtLOX1 and AtLOX3 promoters by an electrophoretic mobility shift assay [56]. The full-length CDS of TaWRKY42-B was subcloned into the frame of pMAL$\mathrm{c} 2 \mathrm{x}$ and fused to the Maltose Binding Protein (MBP). TaWRKY42-B-MBP fusion proteins were expressed and purified from $E$. coli strain Rosetta. Probes against the W-box containing fragments were incubated with TaWRKY42-B-MBP or MBP alone. Probe 1 (P1) and probe 2 (P2) contained the W-boxes of the AtLOX3 promoter, specifically hybridized with TaWRKY42-B-MBP and were competed against unlabeled probes (Fig. 8b). Probe 3 (P3), which was used for testing whether TaWRKY42-MBP targeted AtLOX1, did not show competitive binding with TaWRKY42-B-MBP (Additional file 6: Fig. S6b). These data suggested that AtLOX3 was a potential target gene of TaWRKY42-B in Arabidopsis.

Despite that TaWRKY42-B interacted with AtLOX3, little was known about the potential of TaWRKY42-B binding to the orthologs of $A t L O X 3$ in wheat. We used the AtLOX3 genomic sequence as a query to search against the wheat genome database (http://plants. ensembl.org/Triticum_aestivum). A unique gene (TraesCS4B02G295200), sharing the highest similarity with $A t L O X 3$, was termed TaLOX3 and selected for testing. We searched the TaLOX3 promoter region and found only a single W-box element existing within a $209 \mathrm{bp}$ fragment upstream of the start codon (Fig. 8c). Subsequently, we tested the interaction between TaWRKY42-B and TaLOX3 by EMSA. Consistent with the interaction between TaWRKY42-B and AtLOX3, probe 4 (P4) against the TaLOX3 promoter strongly bound to TaWRKY42-B in vitro (Fig. 8d). To explore the relationship between TaWRKY42-B and TaLOX3 in vivo, the enzymatic activity of LUC, which was under the control of the TaLOX3 promoter and cotransformed with 35S:TaWRKY42-B into wheat protoplasts, was measured (Fig. 8e). The drastic elevation of LUC activity was only observed in protoplasts harboring both 35S:TaWRKY42-B and $P_{\text {TaLOX3:LUC but not in the }}$ control groups (Fig. 8f). Additionally, we analyzed the expression level of TaLOX3 in TaWRKY42-B-silenced plants, and TaLOX3 was remarkably depressed by silencing TaWRKY42-B (Fig. 9a).

Noticeably, the functional role of TaLOX3 was still uncharacterized. Thus, to preliminarily illuminate whether TaLOX3 played a role in JA-induced leaf senescence, we checked the transcripts of TaLOX3 in 7-dayold wheat seedlings, which were treated by $200 \mu \mathrm{M}$ MeJA for $3 \mathrm{~h}$. By the qRT-PCR assay, we illustrated that MeJA was able to enhance the expression of TaLOX3 (Fig. 9b). In addition, decreasing expression of TaLOX3 was detected from the mature leaf stage to the late senescent leaf stage, which is basically in line with the expression pattern of TaWRKY42-B and inverse to the expression of TaSAG3 and TaSAG5 (Fig. 9c-e). Briefly, the above data implied that TaLOX3 is possibly involved in JA-induced leaf senescence in wheat; however, more evidence is still necessary for a comprehensive understanding of the functional role of TaWRKY42-BTaLOX3 module in wheat leaf senescence.

\section{TaWRKY42-B promotes JA biosynthesis}

To further confirm that TaWRKY42-B facilitated leaf senescence via JA biosynthesis, the JA level was measured in young and senescent leaves of TaWRKY42-B$O E$ and wild-type Arabidopsis by LC-MS/MS, respectively. Consistent with previous studies, the JA level was increased throughout the progression of leaf senescence. Notably, the JA level in TaWRKY42-B-OE plants was lower than that in the wild type at the juvenile stage but higher than that of wild-type plants at the senescent stage (Fig. 8g). Based on this data, we speculated that appropriate transcriptional abundance of TaWRKY42-B partially contributed to the homeostasis of JA biosynthesis at different developmental stages, hence the 


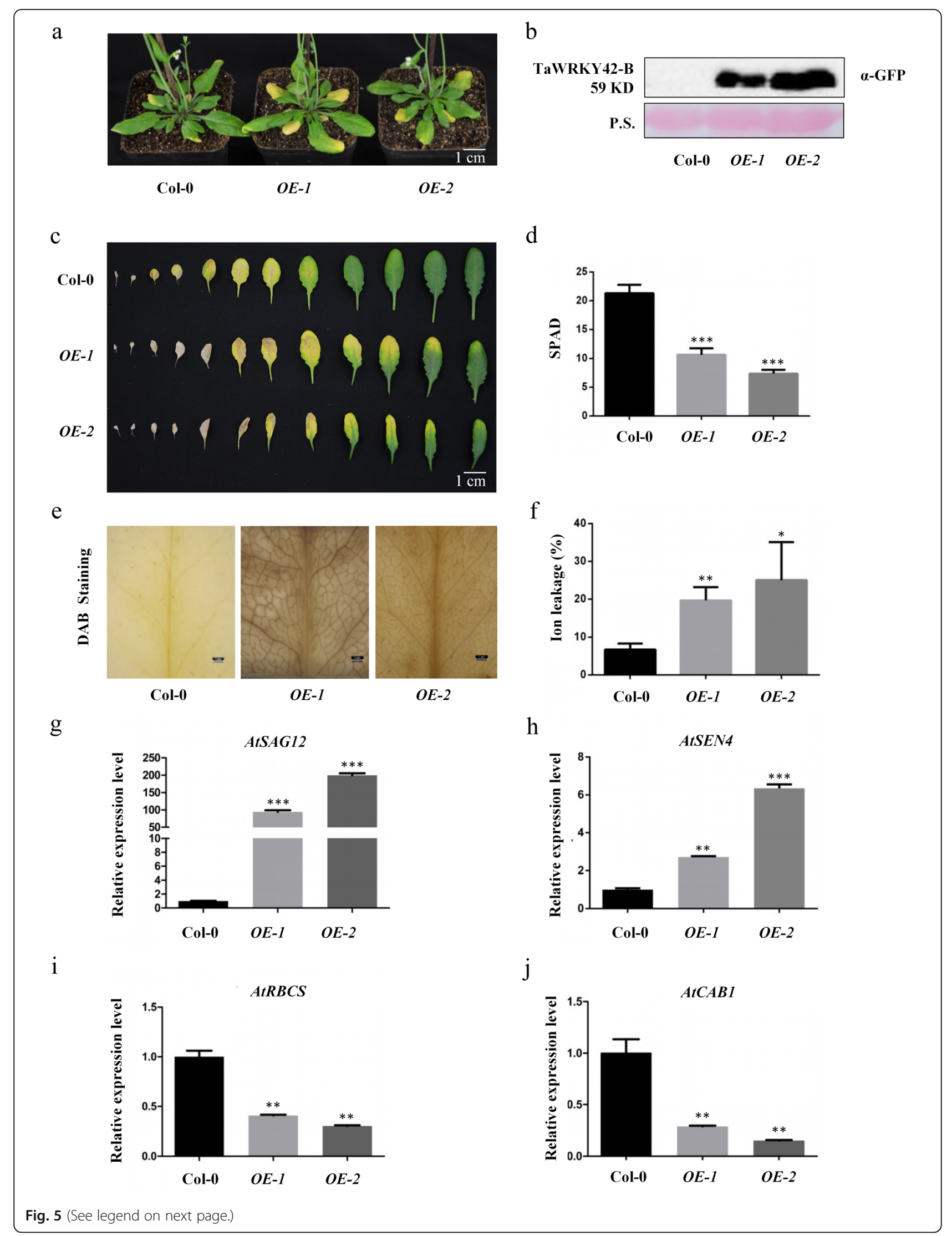


(See figure on previous page.)

Fig. 5 Overexpression of TaWRKY42-B accelerates leaf senescence in Arabidopsis. a Phenotypic analysis of 6-week-old Col-0 and TaWRKY42-Boverexpressing plants. $\mathbf{b}$ Protein level of TaWRKY42-B in Col-0 and TaWRKY42-B-overexpressing plants. Ponceau S (P.S.) staining indicates equal loading. c Detached rosette leaves of 6-week-old Col-0 and transgenic plants were laid out. Chlorophyll content (d) and ion leakage rate (f) of the 6th and 7th detached leaves in (c). e DAB staining of 4-week-old Col-0 and TaWRKY42-B-overexpressing plants. g-j Detection of expression level of senescence-associated genes AtSAG12, AtSEN4, AtRBCS, and AtCAB1 in 4-week-old Col-0 and TaWRKY42-B-overexpressing plants by qRT-PCR. (Error bars indicate SD. Asterisks indicate significant differences. Student's $t$-test, ${ }^{*} P<0.05,{ }^{*} P<0.01,{ }^{* *} P<0.001$ )

expression level of TaWRKY42-B should be tightly controlled.

TaWRKY42-B is conserved with AtWRKY53 in agedependent leaf senescence

AtWRKY53, as a key regulator of the leaf senescence process, integrated various environmental and internal factors for ultimately initiating leaf senescence. In our study, phylogenetic analysis and sequence alignment indicated that TaWRKY42-B was a member of the group III WRKYs, which also comprises AtWRKY53. To validate whether TaWRKY42-B was functionally conserved with AtWRKY53, a complementation experiment was performed, for which CDS of TaWRKY42-B driven by

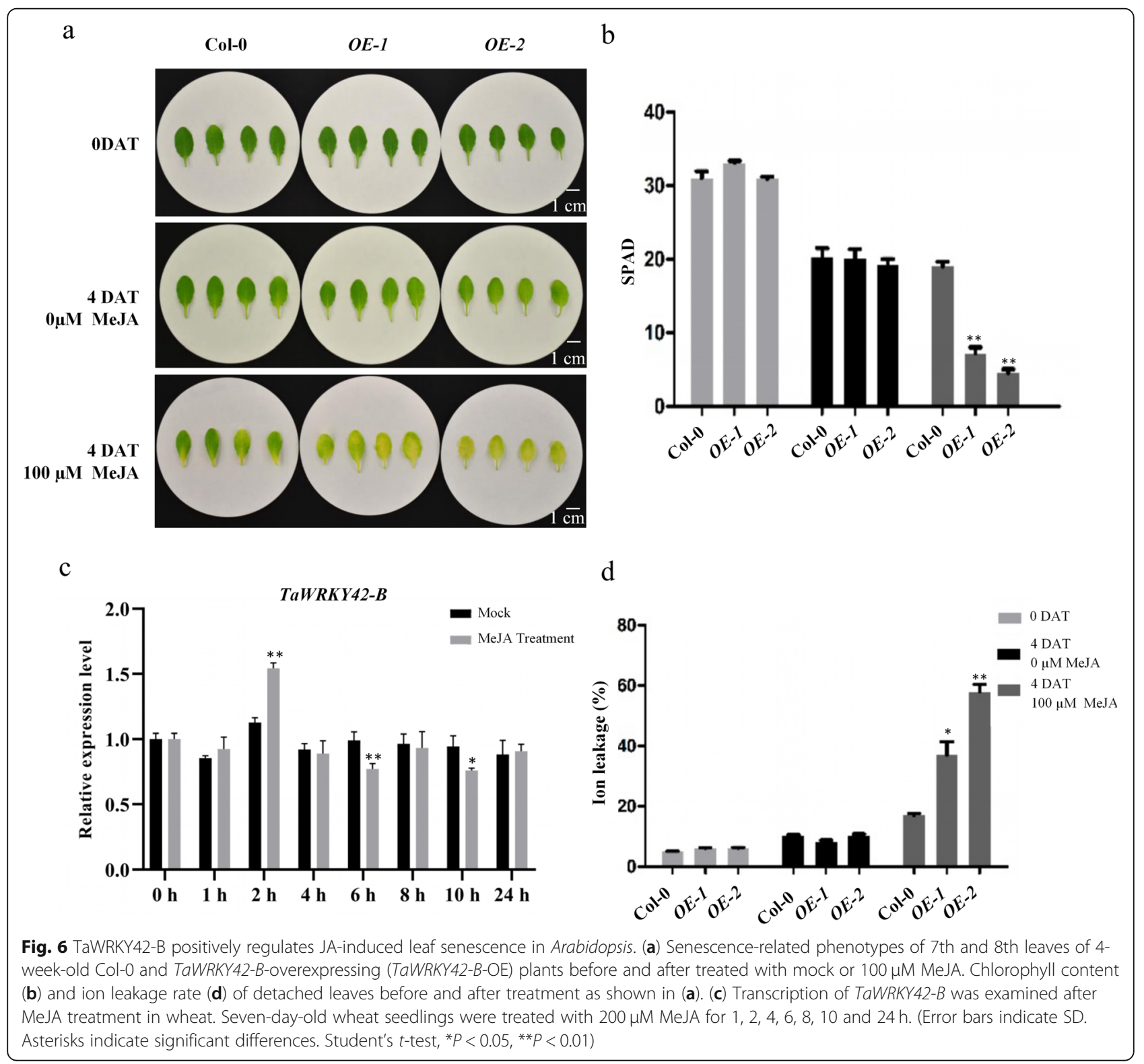




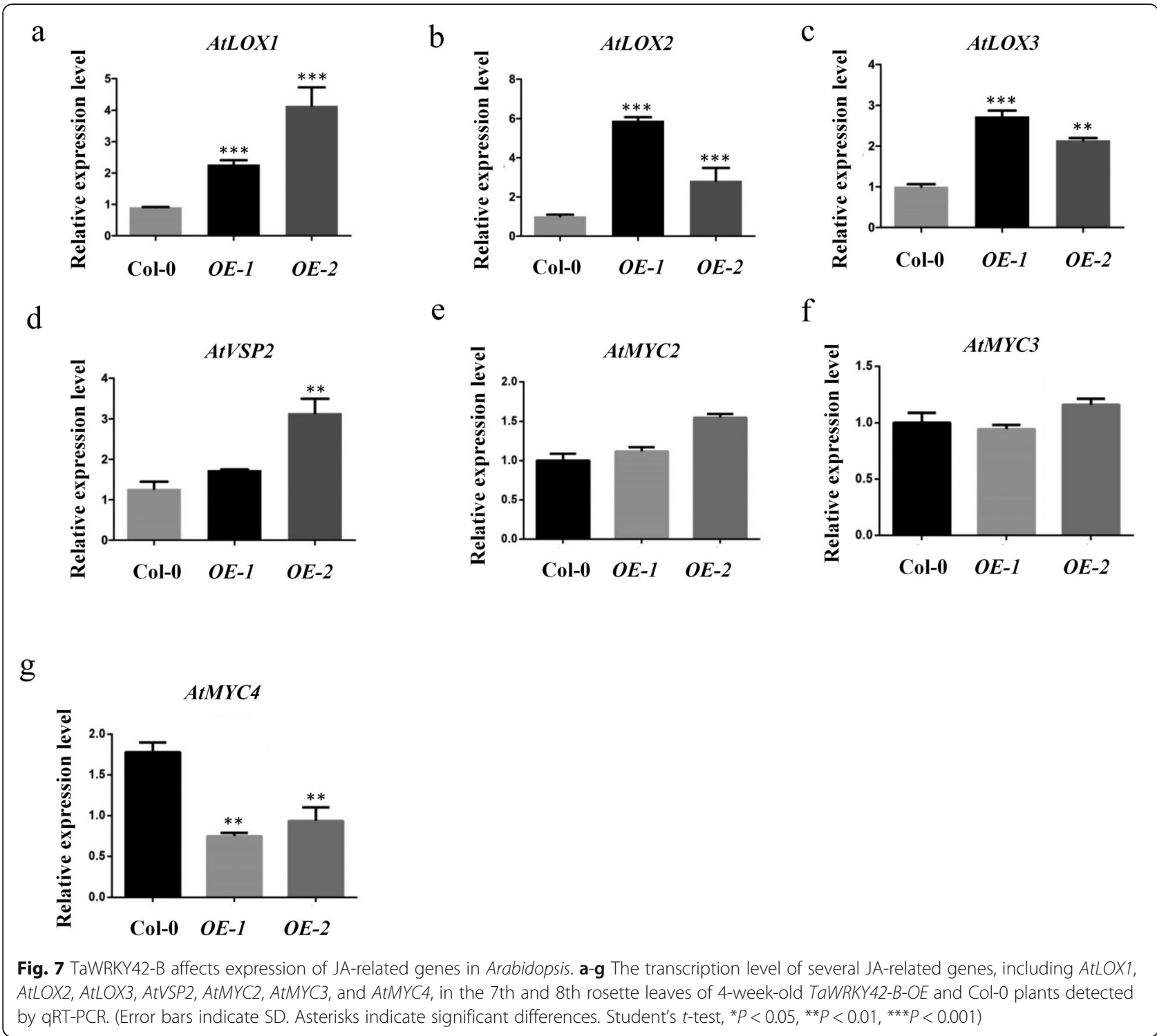

the $1.5 \mathrm{~kb}$ AtWRKY53 promoter was introduced into the atwrky53 mutant (Fig. 10b). The delayed leaf senescence phenotype in atwrky53 was rescued by the expression of TaWRKY42-B (Fig. 10a). Consistently, chlorophyll content, ion leakage rate, and expression level of AtSAG12 and AtRBCS in $P_{A t W R K Y 53}$ TaWRKY42-B/atwrky53 lines was restored (Fig. 10c-f). These data manifested that the functional role of TaWRKY42-B was conserved with AtWRKY53 in the regulation of age-dependent leaf senescence.

\section{Discussion}

Senescence is not only the final developmental stage for plants but is also essential for their productivity and fitness, which has special significance in crops [4]. As leaf senescence proceeds, nitrogen relocates from senescent leaves to grains, which accounts for around $95 \%$ of the total nitrogen in crop grains [57-59]. Improper timing of leaf senescence caused by biotic and abiotic stresses and abnormal developmental events is detrimental to diverse biological processes. Thus, leaf senescence must be a highly ordered program and tightly controlled by a variety of senescence-related genes. The delicate modulation of leaf senescence governed by TFs has been studied for decades and is regarded as a key step for integrating various senescence-related signals.

WRKYs are one of the largest TF families in plants, playing critical roles in developmental regulation and stress defense responses $[39,60]$. Recently, some WRKYs involved biological processes have been studied in wheat. TaWRKY51 affected ethylene production and subsequently promoted lateral root formation [61]. TaWRKY2 was induced by drought, salt, heat, and ABA, and overexpression of TaWRKY2 led to enhanced tolerance of 


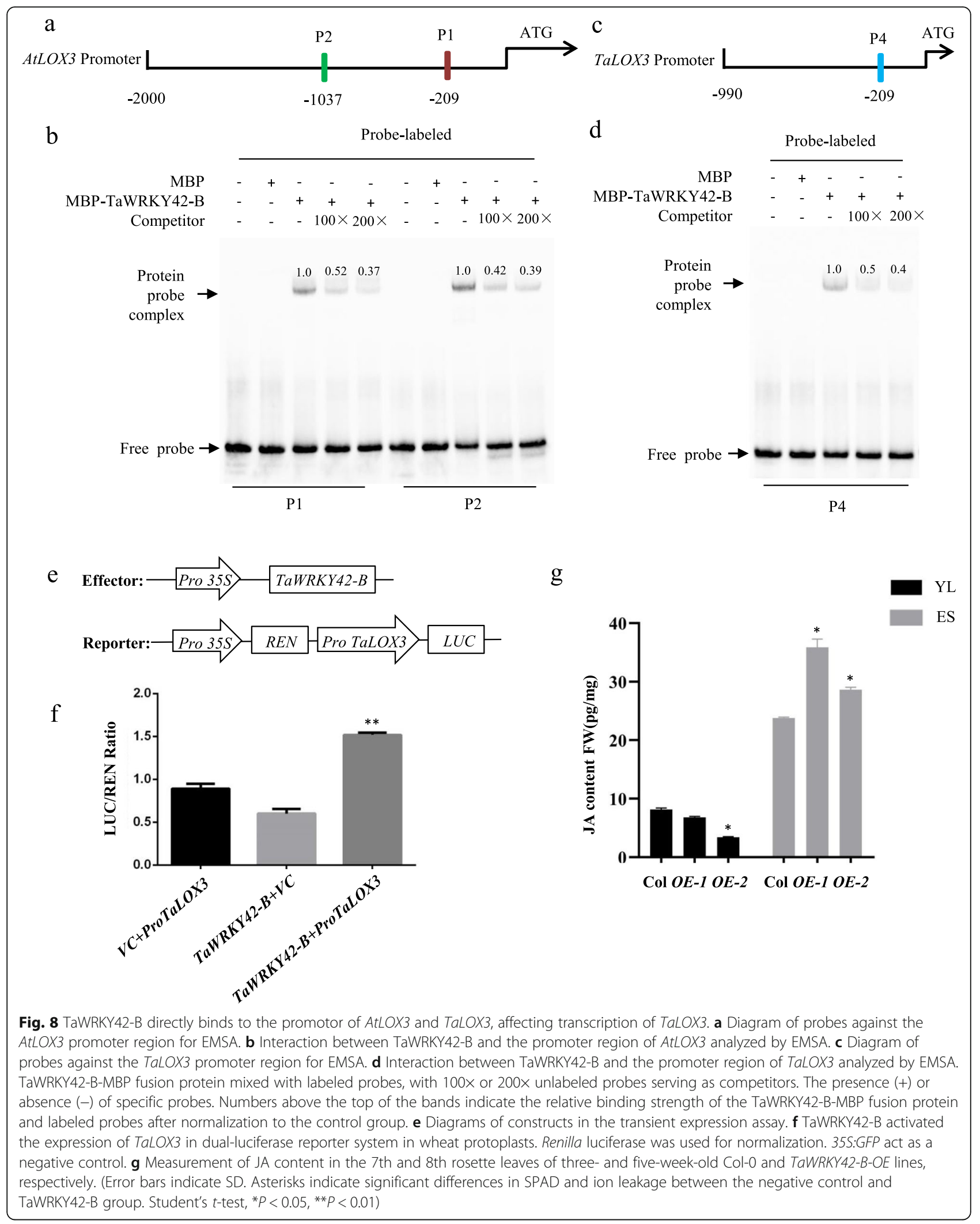




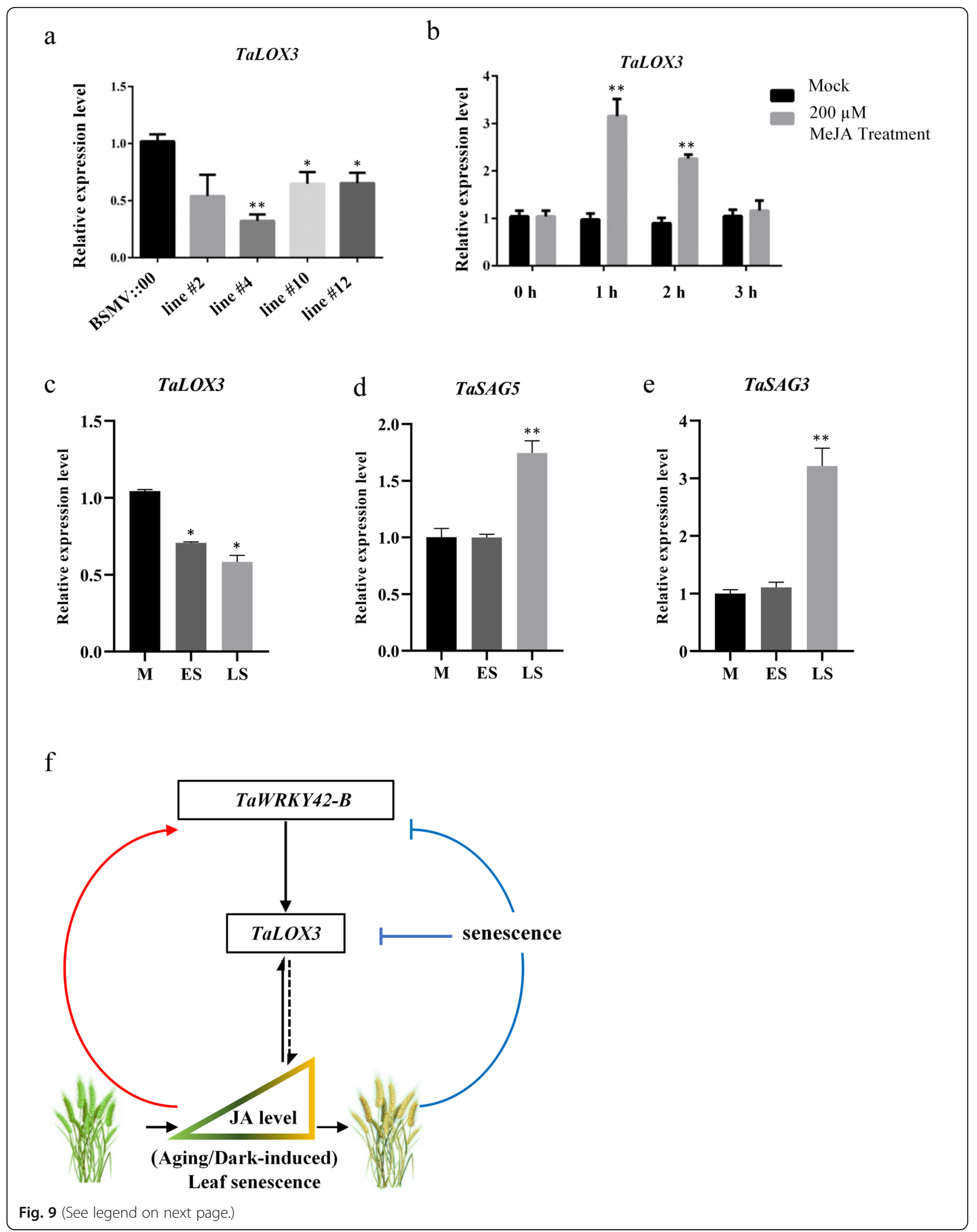


(See figure on previous page.)

Fig. 9 TaLOX3 expression is altered by TaWRKY42-B, MeJA treatment, and the senescence process. a Expression level of TaLOX3 in TaWRKY42-Bsilencing plants. $\mathbf{b}$ Transcription of TaLOX3 was detected after MeJA treatment. Seven-day-old wheat seedlings were treated with $200 \mu \mathrm{M}$ MeJA for 1, 2 and $3 \mathrm{~h}$. c-e Expression pattern of senescence-associated genes TaSAG3, TaSAG5, and TaLOX3 at different developmental stages of wheat flag leaves (M, ES, and LS). $\mathbf{f}$ Proposed model of the TaWRKY42-B-mediated regulatory network of leaf senescence. TaWRKY42-B functions as a promoter of leaf senescence through activating a putative JA biosynthesis gene, TaLOX3, which is also induced by JA signaling. When plants are at the juvenile stage, high levels of TaWRKY42-B are likely responsible for JA-related developmental regulation and biosynthesis. While wheat plants advance into the senescent stage, leaf senescence suppresses the TaWRKY42-B-TaLOX3 module for normal progression of leaf senescence. The feedback control of TaWRKY $42-B$ by JA signaling, and leaf senescence has the potential to be a vital step of timing of leaf senescence. (Error bars indicate SD. Asterisks indicate significant differences. Student's $t$-test, ${ }^{*} P<0.05,{ }^{* *} P<0.01$ )

a

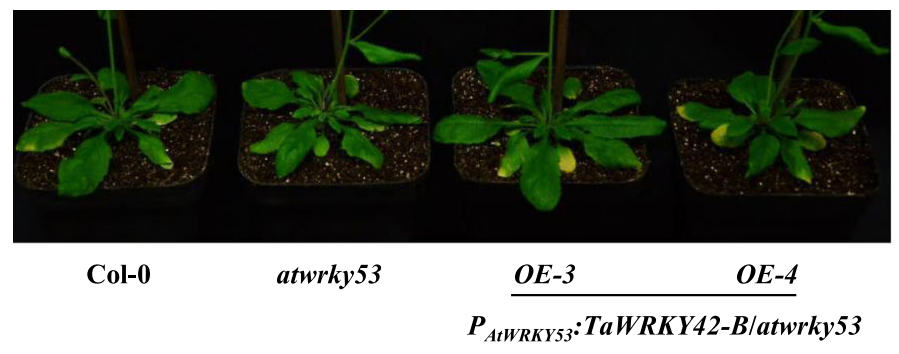

$\mathrm{b}$

TaWRKY42-B 701 bp

AtUBC30

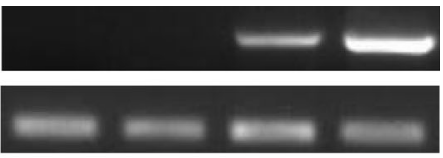

Col-0 atwrky53 OE-3 OE-4

$P_{\text {AtWRKYs3 }}$ TaWRKY42-B/atwrky 53
C

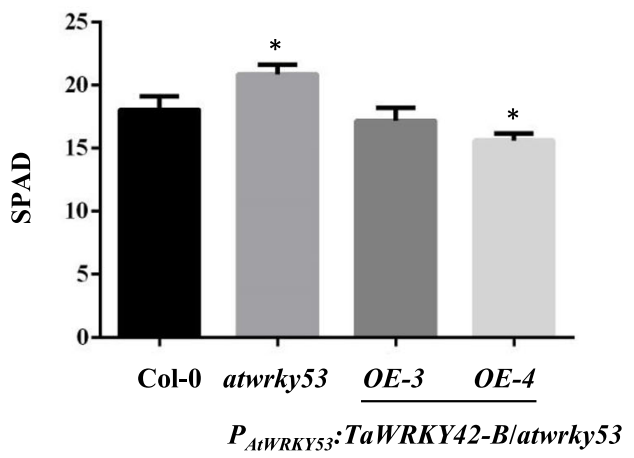

e

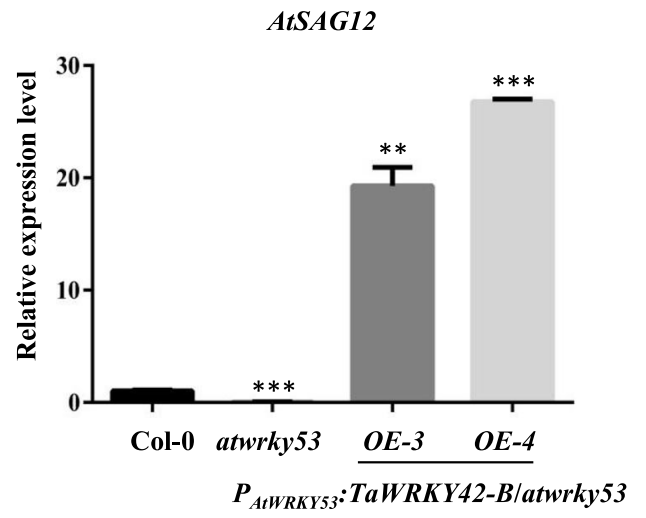

d

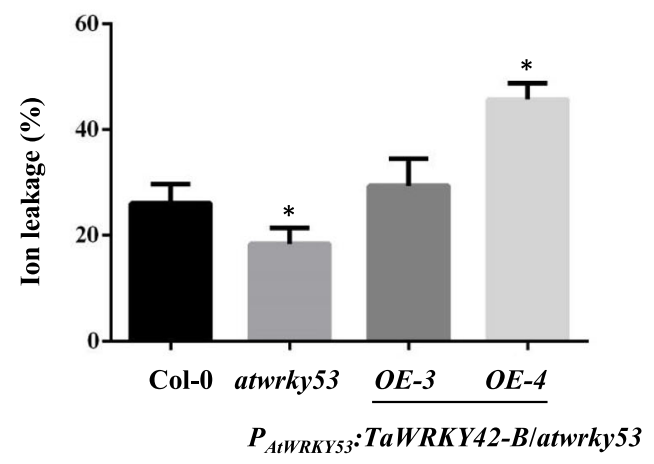

f

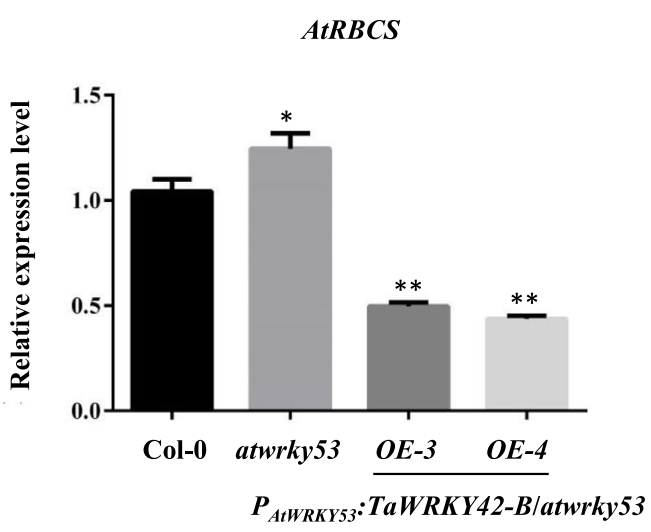

Fig. 10 TaWRKY42-B recovers the delayed leaf senescence phenotype in wrky53. a Senescence-related phenotype of 6-week-old Col-0, atwrky53, and wrky53/WRK42-B-OE lines. b Reverse transcription PCR (RT-PCR) analysis of TaWRKY42-B in PAtwRKY53:TaWRKY42-B/atwrky53, atwrky53, and Col-0 plants. Chlorophyll content (c) and ion leakage rate (d) of different background plants as shown in (a). e-f Transcription level of AtSAG12 and AtRBCS of different background plants as shown in (a). (Error bars indicate SD. Asterisks indicate significant differences. Student's $t$-test, ${ }^{*} P<0.05$, $\left.{ }^{*} P<0.01,{ }^{* *} P<0.001\right)$ 
transgenic wheat to drought stress and increased yield [62, 63]. TaWRKY19 positively regulated salt, drought, and freezing stress responses in Arabidopsis, which was partially due to the elevated expression of DREB2A, $R D 29 A, R D 29 B$, and Cor6.6 by TaWRKY19 [63]. Silencing of TaWRKY70 downregulated the transcription of metabolite biosynthetic genes and thus, decreased the resistance to Fusarium graminearum in wheat [64]. Noticeably, WRKYs are involved in the regulation of leaf senescence in different plant species, but senescencerelated WRKYs are largely unknown in wheat.

In the present study, we screened the leaf senescencerelated TaWRKYs by an RNA-seq experiment. Intriguingly, the transcription level of TaWRKY42-B deceased throughout the progression of leaf senescence, however, TaWRKY42-B played a positive role in the initiation of leaf senescence. This special characteristic of TaWRKY42-B led us to further investigate the functional role of TaWRKY42-B in the regulation of leaf senescence. Similarly, two Arabidopsis regulators of leaf senescence onset, AtWRKY54 and AtWRKY70, are basically accumulated during leaf senescence but they are partially redundant in delaying leaf senescence [47]. Moreover, AtJUB1 delayed leaf senescence initiation and exhibited an age-dependent elevation at the transcriptional level [65]. Previous research partly illustrated that the timing of leaf senescence was orchestrated by a variety of regulators. Thus, the dissection of the sophisticated underlying mechanisms of both senescence-upregulated and -downregulated TFs in the modulation of leaf senescence are of immense importance.

Subsequently, we confirmed the positive role of TaWRKY42-B in the onset of leaf senescence by overexpression of TaWRKY42-B in Arabidopsis. Meanwhile, BSMV-VIGS was a powerful and convenient tool for functional characterization of genes, especially helpful for some crops that are difficult for stable transformation. Using BSMV-VIGS, we found that the onset of leaf senescence was remarkably retarded in wheat plants with a declined expression level of TaWRKY42-B but not TaWRKY42-A and TaWRKY42-D. Notably, following the depression of the mRNA level of TaWRKY42-B upon BSMV-VIGS, transcripts of TaWRKY42-D and TaWRKY42-A presented slight alterations at times, which was possibly led by a compensatory effect and nonspecific silencing. To clarify the connection between TaWRKY42-B and leaf senescence onset in this study, we performed senescence-related phenotypic analyses after detecting the expression the levels of TaWRKY42B, TaWRKY42-A, and TaWRKY42-D in those BSMVVIGS wheat plants. Only the wheat plants with diminished transcripts of TaWRKY $42-B$ and comparable levels of TaWRKY42-A and TaWRKY42-D compared to those of control plants were selected for further analyses.
Thus, we concluded that the delayed leaf senescence phenotype in this study resulted from TaWRKY42-B silencing in wheat. Briefly, the potential of TaWRKY42-B in promoting leaf senescence in monocotyledons and dicotyledons suggested that TaWRKY42-B regulated leaf senescence through a conserved mechanism in plants.

By sequence alignment and phylogenetic analysis, we identified TaWRKY42-B as a member of group III WRKYs, which also comprises some senescence-related WRKYs, such as AtWRKY53. Based on reported studies, senescence-related TFs modulating the initiation of leaf senescence predominately relied on its transcriptional activity. To assess whether TaWRKY42-B impacted the timing of leaf senescence via transcriptional regulation, we examined the transcriptional activity of TaWRKY42$B$ in wheat protoplasts and yeast cells, by which TaWRKY42-B exhibited strong transcriptional activity in both analyses. Consistently, nucleus localization of TaWRKY42-B in wheat mesophyll cell protoplasts also endowed TaWRKY42-B with the potential to regulate the transcription of senescence-related genes.

Furthermore, we demonstrated that TaWRKY42-B affected the leaf senescence process through JA signaling. Leaf senescence was accelerated in TaWRKY42-B-overexpressing plants upon MeJA treatment. Additionally, we confirmed that TaWRKY42-B promoted the accumulation of JA in vivo. Noticeably, ectopic expression of TaWRKY42-B resulted in a lower and a higher JA level at the juvenile and senescent stage, respectively. This result suggested the pleiotropic roles of TaWRKY42-B at different stages. Hence, TaWRKY42-B should be tightly restricted to an appropriate level for normal growth. Consistently, we validated that TaWRKY42-B bound to the promoter regions of AtLOX3 and TaLOX3 by EMSA, which suggested that TaLOX3 and AtLOX3 are potential target genes of TaWRKY42-B. Further, by using dualluciferase reporter system in wheat protoplasts, we demonstrated the activation of TaLOX3 promoter by TaWRKY42-B. Moreover, TaLOX3 showed a decreasing expression level from the mature leaf stage to the late senescent leaf stage, which coincides with the expression pattern of TaWRKY42-B. Collectively, we speculated that the positive correlation between transcription of AtLOX3, TaLOX3, and TaWRKY42-B was a consequence of the interaction of TaWRKY42-B with AtLOX3 and TaLOX3. This modulation was likely promoted by JA accumulation and consequently, triggered the initiation of leaf senescence, although some convincing evidence, such as ChIP-PCR results, will be needed to further validate the physical interaction between TaWRKY42-B and LOXs in future.

JA has long been regarded as a senescence-related phytohormone [31]. Although the content of JA and JA biosynthesis genes are elevated in senescent leaves, the 
senescence process is identical in wild type and some JA-related mutants, which implies that the functional roles of JA signaling in leaf senescence still require consideration [32].

In wheat, a few JA-related genes have been characterized and functionally studied. TaJAZ1 directly interacted with TaABI5, which consequently led to the inhibition of seed germination [66]. TaJAZ1 also positively regulated the resistance to powdery mildew [67]. TaAOC1, a JA biosynthesis gene, was responsible for JA accumulation and tolerance to salinity [68]. TaOPR2 functioned in JA biosynthesis and could rescue the male sterility phenotype of opr3 in Arabidopsis [69]. However, identification of the regulators involved in JA-mediated leaf senescence in wheat is lagging behind many crops. The dramatically delayed leaf senescence phenotype of TaWRKY42-B-silenced wheat plants and precocious leaf senescence in TaWRKY42-B-overexpressing Arabidopsis manifested that TaWRKY42-B had a positive role in the onset of leaf senescence. Our data also suggested that TaWRKY42-B promoted leaf senescence via the JA pathway. Intriguingly, TaWRKY42-B was a promoter of the leaf senescence onset but with a decreasing expression pattern as leaf senescence proceeded. To explain the functional role of TaWRKY42-B, we proposed a hypothesis that is mainly based on the effect of TaWRKY42-B on JA biosynthesis in leaf senescence. We speculated that TaWRKY42-B and some of the JA biosynthesisrelated genes were under tight control to maintain a proper content of JA for normal growth in wheat. Before the initiation of leaf senescence, TaWRKY42-B exhibited a relatively high expression level at the juvenile stage for JA accumulation and JA-related developmental regulations, including preparation for the initiation of leaf senescence, which in turn activated TaWRKY42-B expression. As leaf senescence progressed, TaWRKY42-B and its targets, such as TaLOX3, underwent continuous suppression and cooperated with other senescencerelated regulators for organized progression of leaf senescence. It is undeniable that JA content is almost continuously elevated with the progression of senescence, suggesting that accumulation of JA during leaf senescence is governed by other regulators. However, some detailed information on the underlying mechanism of the TaWRKY42-B-TaLOX3 module and some in vivo experiments are needed to further validate our hypothesis.

Finally, based on our genetic data, we manifested that TaWRKY42-B involved leaf senescence regulation was partially conserved with AtWRKY53. Full-length TaWRKY42-B CDS driven by the AtWRKY53 promoter was able to rescue delayed leaf senescence in atwrky53. Previously, experimental data showed that AtWRKY53 was antagonistically regulated by JA and SA signaling in leaf senescence. In our study, we discovered that TaWRKY42-B modulating leaf senescence was also connected with JA, which further implied that TaWRKY42B was functionally conserved with AtWRKY53 in agedependent leaf senescence.

Briefly, we proposed a model of TaWRKY42-Bpromoted leaf senescence (Fig. 9f). The transcription level of TaWRKY42-B was tightly controlled by the progression of leaf senescence and JA signaling. In nonsenescent leaves, increased JA active TaWRKY42-B triggered the transcription of TaLOX3 and subsequently, elevated JA biosynthesis for leaf senescence onset. However, at the senescence stage, TaWRKY42-BTaLOX3 was suppressed for organized leaf senescence progression. This feedback loop was likely to contribute to the proper timing of leaf senescence in wheat. Being functionally conserved with AtWRKY53, TaWRKY42-B was likewise involved in JA-related leaf senescence and altered the expression of some JA biosynthesis genes, such as AtLOX3 and TaLOX3. Although more in vivo evidence is needed for comprehensive understanding of the functional role of TaWRKY42-B, we shed a light on the detailed information about the transcriptional regulation of leaf senescence in wheat.

\section{Conclusions}

Collectively, by physiological and molecular analyses, we identified a novel WRKY-type promoter of leaf senescence, TaWRKY42-B, which mediated leaf senescence initiation by promoting JA biosynthesis and interacting with AtLOX3 and TaLOX3. Our results provide a new clue for uncovering the mechanisms underlying wheat leaf senescence and a potential target gene for improving productivity and quality through modulating the leaf senescence process in wheat.

\section{Methods}

Plant materials and growth conditions

Arabidopsis thaliana ecotype 'Col-0' was obtained from the Arabidopsis Biological Resource Center (ABRC; http://abrc.osu.edu) and used for transformation. Arabidopsis mutant atwrky53 (SALK_034157) was kindly provided by Prof. Ying Miao (Fujian Agriculture and Forestry University) and used for the complementation experiment. The atwrky53 mutant had a T-DNA insertion within the second exon and genotyping of atwrky53 in this study was described previously [41]. The delayed leaf senescence phenotype of atwrky53 was also confirmed by authors through the measurement of senescence-related parameters. All Arabidopsis plants were grown in a growth chamber at Hebei Normal University. Surface-sterilized seeds were sown in $1 / 2 \mathrm{MS}$ medium containing $1 \%$ sucrose, $0.8 \%$ agar, and $1 \times$ Murashige and Skoog salt, and treated at $4{ }^{\circ} \mathrm{C}$ for $48 \mathrm{~h}$. 
Seven-day-old seedlings were transferred to the green house at $22^{\circ} \mathrm{C}$ under long-day conditions (LD: $16 \mathrm{~h}$ light /8 h dark). Four-week-old plants grown in LD condition were treated with darkness and MeJA, and chlorophyll content and ion leakage were measured.

The bread wheat cultivar "ShiLuan 02-1" is widely cultivated for agricultural production in China. In this study, "ShiLuan 02-1" was kindly provided by Prof. Zhanjing Huang (Hebei Normal University) and used for BSMV-VIGS, and detection of TaWRKY42-B expression pattern. Seeds of "ShiLuan 02-1" were preserved in our lab and could also be obtained from the seed bank of the Institute of Genetics and Physiology, Hebei Academy of Agriculture and Forestry Sciences. Phenotypic characters and agronomic traits of "ShiLuan 02-1" were analyzed by the authors for the confirmation of the "ShiLuan 02-1" background. Wheat plants were grown under greenhouse conditions at Hebei Normal University and Hebei Academy of Agriculture and Forestry Sciences. Seven-day-old seedlings were used for infiltration. Five-week-old infiltrated plants were treated with darkness and the expression level of TaWRY42-B and SAGs was detected. For examining the expression level of TaWRKY42-B at four developmental leaf stages, wheat flag leaves were harvested from April to June; and the timing of collection was described in Arabidopsis based on the chlorophyll content, ion leakage rate, and expression level of SAGs [22]. The bread wheat cultivar "KeNong 199" was used to generate wheat protoplasts.

\section{Plasmid construction and plant transformation}

For the overexpression of TaWRKY42-B, the full-length $879 \mathrm{bp}$ CDS of TaWRKY42-B was amplified from the flag leaves of wheat and introduced to the pCAMBIA1300-GFP vector. The above vector was transferred into Agrobacterium strain GV3101 and transformed to Arabidopsis through the floral dip transformation method. TaWRKY42-B-GFP fusions in transgenic lines were detected by the western-blot assay with a GFP antibody.

To test transcriptional activity and subcellular localization, TaWRKY42-B was subcloned into pSATGAL4DB, pGBKT7, and PUC19 vectors.

For EMSA, full-length cDNA of TaWRKY42-B was generated and cloned into the pMAL-C2X vector and the construct was transformed into E. coli strain Rosetta.

To generate TaWRKY42-B BSMV-VIGS constructs, a 198 bp fragment of TaWRKY42-B was constructed into the pCaBS- $\gamma$ bLIC vector.

\section{Protoplast transformation and dual-luciferase reporter assay}

Protoplasts were isolated from 7-day-old etiolated "KeNong 199" seedlings. pGreen0800-LUC was fused with the TaLOX3 promoter and used as a reporter. 35S: TaWRKY42-B-GFP was used as an effector and 35S:GFP as a negative control. The plasmids pGreen0800-LUC and 35S:TaWRKY42-B-GFP or 35S:GFP, were cotransformed into wheat protoplasts and incubated for $18 \mathrm{~h}$. The firefly and Renilla luciferase activity were quantified by the dual-luciferase assay kit (Promega, E1910).

\section{Ion leakage and chlorophyll content}

The relative chlorophyll content of rosette leaves was detected with the SPAD502 Plus Chlorophyll Meter. For measurement of membrane ion leakage, 7th leaf of each plant was harvested and placed in deionized water under vacuum for $1 \mathrm{~h}$. Conductivity of different background leaves were measured. Next, the conductivity of leaves which were boiled in deionized water for $15 \mathrm{~min}$ were measured and used as total conductivity. After subtracting the conductivity value of water, conductivity of leaves before and after boiling was used to generate the value of ion leakage rate. The ratio of conductivity of leaves before and after boiling was finally identified as ion leakage rate [19].

\section{qRT-PCR}

Total RNA was extracted using the Trizol reagent (TAKARA, 9109). Total RNA (500 ng) was treated with $1 \mu \mathrm{L}$ DNase I and used for preparing the first-strand cDNA. Synthesized cDNA was quantified by qRT-PCR. Real-time PCR was performed by mixing SYBR green (TAKARA, RR420A) with the ROX reference dye in ABI7300. Each sample was analyzed in three biological replicates. All the primers used in this study were listed in Additional file 7: Table S1. The expression levels of target genes were normalized to the expression level of the internal control, TaACTIN in wheat, and the expression levels were normalized to the expression level of the internal control, AtUBC30 in Arabidopsis.

\section{RNA-Seq}

Total RNA was isolated from Chinese Spring flag leaf blades at booting, heading, Anthesis and Grain-filling stage using TIANGEN ${ }^{\circ}$ RNAprep Pure Plant Plus Kit (Polysaccharides\&Polyphenolics-rich). Three biological replicates were employed. NEBNext ${ }^{\circ}$ Ultra $^{\mathrm{Tw}}$ RNA Library Prep Kit for Illumina ${ }^{\circ}$ (NEB, USA) was used to generate non-stranded sequencing libraries according to instructions. TruSeq PE Cluster Kit v3-cBot-HS (Illumina) was used to cluster the index-coded samples by the cBot Cluster Generation System according to the manufacturer's recommendations. Next, Illumina Novaseq6000 platform was used to perform the sequencing of the libraries, therefore the reads with $150 \mathrm{bp}$ paired-end were generated. The overall sequencing quality of the reads in 
each sample was evaluated and controlled with the software Fastp by using default parameters [70]. The remaining reads were aligned to the wheat reference genome IWGSCv1.0 (http://www.wheatgenome.org/) using HISAT2 v2.1 (https://daehwankimlab.github.io/ hisat2/) with the default parameters, and only the uniquely mapped reads were retained for the following analysis (Additional file 8: Table S2). The gene expression quantification was done by HTseq [71, 72]. The $\mathrm{R}$ package DESeq2 was used to perform differential expression analysis between each two stages, and only the genes with an absolute value of $\log _{2}$ (fold change) $\geq 1$ and $P$ value $<0.05$ were considered as DEGs.

The RNA sequencing data have been deposited into NCBI Sequence Read Archive (https://www.ncbi.nlm. nih.gov/bioproject/PRJNA656068) under the accession no. PRJNA656068 and CNSA (https://db.cngb.org/ search/project/CNP0001003) with the accession no. CNP0001003.

\section{Phylogenetic analysis and gene expression}

The phylogenetic tree was constructed by MEGA X using the maximum likelihood method with WAG+F model [73] and outputted as a Newick file, which was uploaded to the Interactive Tree Of Life (https://itol. embl.de/), an online tool for tree design, to create the figure. The gene expression levels were based on FPKM values from RNA-Seq data and standardized with $\log 2(\mathrm{FPKM}+1)$. The heatmap was constructed with TBtools [74].

\section{Dark-induced leaf senescence}

For dark-induced leaf senescence, One-month-old wheat leaves and 6th and 7th rosette leaves of 4-week-old Arabidopsis plants were placed into dishes with $10 \mathrm{~mL}$ deionized water. The samples were kept in the dark for 5 or 6 days, and then the chlorophyll content and the ion leakage were measured.

For JA-induced leaf senescence, the 6th and 7th leaves were treated with deionized water or $100 \mu \mathrm{M} \mathrm{MeJA}$ solution and the samples were kept in the dark at $22^{\circ} \mathrm{C}$ for 4 or 5 days. Then the chlorophyll content and ion leakage were measured.

\section{DAB staining}

Deionized water was adjusted to a pH of 3 with $\mathrm{HCl}, 1 \%$ $\mathrm{DAB}$ was added until completely dissolved, and then, the $\mathrm{pH}$ was adjusted to 5.7 with $\mathrm{KOH}$. The detached leaves of Arabidopsis and wheat were immersed in 1\% $\mathrm{DAB}$ solution and under a vacuum condition for $15 \mathrm{~min}$. Next, above leaves were incubated in darkness for another $4-6 \mathrm{~h}$ at room temperature. Samples were placed in $75 \%$ alcohol until discolored.

\section{BSMV}

The target fragment of TaWRKY42-B was cloned into the pCaBS- $\gamma$ bLIC plasmid by the LIC strategy and equal amounts of bacteria harboring pCaBS- $\alpha, \mathrm{pCaBS}-\beta$, and pCaBS- $\gamma$ bLIC were mixed and infiltrated into $N$. benthamiana leaves. The solution contained $10 \mathrm{mM}$ $\mathrm{MgCl}_{2}, 10 \mathrm{mM}$ MES, and $0.1 \mathrm{M}$ Acetyleugenone $(\mathrm{pH}=$ 5.2). Seven days after infiltration, the infected local leaves were harvest for the sap and the sap was mechanically inoculated onto wheat at the two-leaf stage. The presence of the vector BSMV::TaWRKY42- $\mathrm{B}_{198}$ in $N$. benthamiana could be detected after 5 days of infection, which indicated successful infection. In addition, BSMV:: TaPDS, as the positive control, and the empty (pCaBS- $\alpha$, pCaBS- $\beta$ and pCaBS- $\gamma$ bLIC) as negative controls.

\section{JA quantification}

Three-week-old and 5-week-old TaWRKY42-B-OE and Col-0 Arabidopsis plants for JA analysis were processed and purified as previously described [75]. Detached leaves $(200 \mathrm{mg}$ ) of TaWRKY42-B-OE and Col-0 plants were homogenized and immersed in methanol for $24 \mathrm{~h}$. After centrifugation of all samples, purification was conducted by using the Oasis Max solid phase extract cartridge. JA content of all samples were measured by UPLC system (Waters) and QTRAP 5500 system (AB SCIEX). Three biological replicates were performed and ${ }^{2} \mathrm{H}_{5}$-JA was used as the internal reference standard.

\section{EMSA}

For EMSA, the TaWRKY42-B protein fused with MBP was expressed in E. coli (Rosetta) and purified with Amylose Resin (0812S, New England Biolabs, USA). Expression of the TaWRKY42-B-MBP fusion protein was induced in $200 \mathrm{~mL}$ cultures of transformed bacteria by the addition of IPTG to a final concentration of $0.5 \mathrm{mM}$; and cultures were incubated at $25^{\circ} \mathrm{C}$ for $6 \mathrm{~h}$. Biotinlabeled DNA probes are listed in Additional file 7: Table S1. Unlabeled competitor probes were added at 100 -fold and 200 -fold molar excess. EMSA was performed by using the Chemiluminescent Nucleic Acid Detection Module (Thermo Scientific, 89,880). A total reaction volume of $10 \mu \mathrm{L}$ contained $1 \mu \mathrm{L}$ binding buffer, $0.5 \mu \mathrm{L}$ glycerol, $0.5 \mu \mathrm{L}$ poly-dIdC, $1 \mu \mathrm{L}$ biotin-probe, $0.5 \mu \mathrm{L} 1 \mathrm{M}$ $\mathrm{KCl}$, and $400 \mathrm{ng}$ purified fusion protein. Biotin-labeled DNA was detected using the Stable Peroxide Solution (Thermo Scientific, 89,880).

\section{Supplementary information}

Supplementary information accompanies this paper at https://doi.org/10. 1186/s12870-020-02650-7.

Additional file 1: Figure S1. Sequence alignment of TaWRKY42-B TaWRKY42-A, and TaWRKY42-D. (a) Cluster analysis among WRKY 
subgroups and TaWRKY42-B. (b) Alignment of nucleotide sequences of TaWRKY42-B, TaWRKY42-A, and TaWRKY42-D. (c) Alignment of TaWRKY42B, TaWRKY42-A, and TaWRKY42-D protein sequences in DNAMAN.

Additional file 2: Figure S2. The target fragment of TaWRKY $42-B$ for BSMV-VIGS. (a) Sequence of the target fragment of TaWRKY42-B in BSMVVIGS. (b) Detection of BSMV::TaWRKY42-B 198 vector in infiltrated $N$. benthamiana. BSMV::00 was used as a negative control and BSMV::TaWRKY42-B B $_{198}$ plasmid served as a positive control (PC). Lane 1 and 2 showed the PCR produces of BSMV::TaWRKY42-B198 vector in two infiltrated leaves, respectively.

Additional file 3: Figure S3. TaWRKY42-B possesses transcriptional activity in yeast. (a) Yeast strains containing the indicated vectors were grown on SD/-Trp-His-Leu medium. The strain contained the pGBKT7 vector as the negative control. TaNAC6 and TaWRKY5-D were fused to pGBKT7 as the positive control. (b) The above yeast colonies were analyzed by X-gal staining.

Additional file 4: Figure S4. Promoter analysis of TaWRKY 42-B in PlantCARE. The localization of cis-elements in TaWRKY42-B promoter region. The CGTCA-motif was involved in MeJA responsiveness, TCAelement was involved in SA responsiveness, and ABRE was involved in ABA responsiveness.

Additional file 5: Figure S5. Overexpression of TaWRKY $42-B$ promotes dark-induced leaf senescence. (a) Detached leaves of 4-week-old Col-0 and TaWRKY42-B-overexpressing plants were treated with darkness for 6 days. Chlorophyll content (b) and ion leakage rate (c) of detached leaves before and after dark treatment as shown in (a). (Error bars indicate SD. Asterisks indicate significant differences in SPAD and ion leakage between TaWRKY42-B-OE lines and Col-0. Student's $t$-test, ${ }^{*} P<0.05$, ${ }^{* *} P<$ 0.01).

Additional file 6: Figure S6. TaWRKY42-B cannot directly bind to the promotor of AtLOX1. (a) Diagram of probes against AtLOX1 promoter region for EMSA. (b) The interaction between TaWRKY42-B and promoter region of AtLOX1 was analyzed by EMSA. TaWRKY42-B-MBP fusion protein mixed with labeled probes and 100x or 200x unlabeled probes served as competitors. The presence $(+)$ or absence $(-)$ of specific probes existence or not. Numbers above the top of the bands indicate the relative binding strength of the TaWRKY42-B-MBP fusion protein and labeled probes after normalization to the control group.

Additional file 7: Table S1. Primers and probes used in this study.

Additional file 8: Table S2. Number of reads in each library.

\section{Abbreviations}

ABA: Abscisic acid; AUXs: Auxins; BRs: Brassinosteroids; BSMV: Barley stripe mosaic virus; CKs: Cytokinins; DAB: 3,3-diaminobenzidine; DAT: Day after treatment; EMSA: Electrophoretic mobility shift assay; GA: Gibberellic acid; JA: Jasmonic acid; LUC: Firefly luciferase; MeJA: Methyl jasmonate: SA: Salicylic acid; SAGs: Senescence-associated genes; SLs: Strigolactones; REN: Renilla luciferase; TF: Transcription factor; VIGS: Virus-induced gene silencing; WT: Wild type

\section{Acknowledgements}

We thank Prof. Dawei Li for sharing BSMV vectors and Prof. Ying Miao for sharing atwrky53 mutant. We also thank Prof. Zhanjing Huang for providing wheat cultivar "ShiLuan 02-1".

\section{Authors' contributions}

$\mathrm{CZ}$ and $\mathrm{GW}$ conceived and designed the experiments. MZ, XZ, and QT generated the constructs and transgenic lines. $\mathrm{MZ}, \mathrm{XZ}, \mathrm{YL}$, and $\mathrm{SZ}$ tested the transcriptional activity and performed the phenotypic and physiological analysis. KL conducted the bioinformatics analysis. MZ performed the EMSA experiment. MZ, GW, and CZ wrote the manuscript. All authors read and approved the final manuscript.

\section{Funding}

This work was supported by grants from National Natural Science Foundation of China (Grant No. 31671694 and 31871616 to CZ, Grant No. 31600227 and 31970199 to GW), Major Basic Research Program of Hebei Province (Grant No. 18966337D to CZ), HAAFS Agriculture Science and
Technology Innovation Project (Grant No. 2019-4-8-1 to GW) and China Postdoctoral Science Foundation (Grant No. 2017 M621096 to GW). The funders had no role in the design of the study and collection, analysis, and interpretation of data and in writing the manuscript.

\section{Availability of data and materials}

RNA-seq data has been deposited into CNSA (https://db.cngb.org/search/ project/CNP0001003) with accession no. CNP0001003 and NCBI Sequence Read Archive (https://www.ncbi.nlm.nih.gov/bioproject/PRJNA656068) under the accession no. PRJNA656068. All the other experimental data about in this study are presented in this article and its supplementary information files.

\section{Ethics approval and consent to participate}

Not applicable.

\section{Consent for publication}

Not applicable.

\section{Competing interests}

The authors declared that they have no competing interest.

\section{Author details}

${ }^{1}$ Ministry of Education Key Laboratory of Molecular and Cell Biology, Hebei Collaboration Innovation Center for Cell Signaling, College of Life Sciences, Hebei Normal University, Shijiazhuang 050024, Hebei, China. ${ }^{2}$ Institute of Genetics and Physiology, Hebei Academy of Agriculture and Forestry Sciences /Plant Genetic Engineering Center of Hebei Province, Shijiazhuang 050051, Hebei, China.

Received: 2 December 2019 Accepted: 15 September 2020

Published online: 29 September 2020

\section{References}

1. Thomas H, Stoddart JL. Leaf senescence. Ann Rev Plant Physiol. 1980;31:83111.

2. LDN. The phenomena of senescence and aging. In: Nooden LD, editor. Senescence and aging in plants. San Diego: Elsevier Academic Press; 1988. p. 1-50.

3. Lim PO, Kim HJ, Nam HG. Leaf senescence. Annu Rev Plant Biol. 2007;58: 115-36.

4. Gregersen PL, Culetic A, Boschian L, Krupinska K. Plant senescence and crop productivity. Plant Mol Biol. 2013;82(6):603-22.

5. Woo HR, Kim HJ, Lim PO, Nam HG. Leaf senescence: systems and dynamics aspects. Annu Rev Plant Biol. 2019:70:347-76.

6. Liu C, Cheng J, Zhuang Y, Ye L, Li Z, Wang Y, Qi M, Xu L, Zhang Y. Polycomb repressive complex 2 attenuates ABA-induced senescence in Arabidopsis. Plant J. 2019;97(2):368-77.

7. Wang X, Gao J, Gao S, Song Y, Yang Z, Kuai B. The H3K27me3 demethylase REF6 promotes leaf senescence through directly activating major senescence regulatory and functional genes in Arabidopsis. PLoS Genet. 2019;15(4):e1008068

8. Liu P, Zhang S, Zhou B, Luo X, Zhou XF, Cai B, Jin YH, Niu LJ, Cao X, et al. The histone H3K4 Demethylase JMJ16 represses leaf senescence in Arabidopsis. Plant Cell. 2019;31(2):430-43.

9. Kim H, Kim HJ, Vu QT, Jung S, McClung CR, Hong S, Nam HG. Circadian control of ORE1 by PRR9 positively regulates leaf senescence in Arabidopsis. Proc Natl Acad Sci U S A. 2018;115(33):8448-53.

10. Zhang Y, Wang Y, Wei H, Li N, Tian W, Chong K, Wang L. Circadian evening complex represses Jasmonate-induced leaf senescence in Arabidopsis. Mol Plant. 2018;11(2):326-37.

11. He L, Wu W, Zinta G, Yang L, Wang D, Liu R, Zhang H, Zheng Z, Huang H, Zhang $\mathrm{Q}$, et al. A naturally occurring epiallele associates with leaf senescence and local climate adaptation in Arabidopsis accessions. Nat Commun. 2018;9(1):460

12. Jia M, Liu X, Xue H, Wu Y, Shi L, Wang R, Chen Y, Xu N, Zhao J, Shao J, et al. Noncanonical ATG8-ABS3 interaction controls senescence in plants. Nat Plants. 2019;5(2):212-24.

13. Springer A, Kang C, Rustgi S, von Wettstein D, Reinbothe C, Pollmann S, Reinbothe S. Programmed chloroplast destruction during leaf senescence involves 13-lipoxygenase (13-LOX). Proc Natl Acad Sci U S A. 2016;113(12): $3383-8$ 
14. Li Z, Wu S, Chen J, Wang X, Gao J, Ren G, Kuai B. NYEs/SGRs-mediated chlorophyll degradation is critical for detoxification during seed maturation in Arabidopsis. Plant J. 2017;92(4):650-61.

15. Schippers $\mathrm{JH}$. Transcriptional networks in leaf senescence. Curr Opin Plant Biol. 2015;27:77-83.

16. Penfold CA, Buchanan-Wollaston V. Modelling transcriptional networks in leaf senescence. J Exp Bot. 2014;65(14):3859-73.

17. Yuan L, Wang D, Cao L, Yu N, Liu K, Guo Y, Gan S, Chen L. Regulation of leaf longevity by DML3-mediated DNA Demethylation. Mol Plant. 2020;13(8): 1149-61.

18. Schippers JH, Schmidt R, Wagstaff C, Jing HC. Living to die and dying to live: the survival strategy behind leaf senescence. Plant Physiol. 2015;169(2):914-30.

19. Li Z, Peng J, Wen X, Guo H. Ethylene-insensitive3 is a senescence-associated gene that accelerates age-dependent leaf senescence by directly repressing miR164 transcription in Arabidopsis. Plant Cell. 2013;25(9):3311-28.

20. Zhao Y, Chan Z, Gao J, Xing L, Cao M, Yu C, Hu Y, You J, Shi H, Zhu Y, et al. ABA receptor PYL9 promotes drought resistance and leaf senescence. Proc Natl Acad Sci U S A. 2016;113(7):1949-54.

21. Qi T, Wang J, Huang H, Liu B, Gao H, Liu Y, Song S, Xie D. Regulation of Jasmonate-induced leaf senescence by antagonism between bHLH subgroup IIIe and IIId factors in Arabidopsis. Plant Cell. 2015;27(6):1634-49.

22. Guo P, Li Z, Huang P, Li B, Fang S, Chu J, Guo H. A tripartite amplification loop involving the transcription factor WRKY75, salicylic acid, and reactive oxygen species accelerates leaf senescence. Plant Cell. 2017;29(11):2854-70.

23. Sun Y, Fan XY, Cao DM, Tang W, He K, Zhu JY, He JX, Bai MY, Zhu S, Oh E, et al. Integration of brassinosteroid signal transduction with the transcription network for plant growth regulation in Arabidopsis. Dev Cell. 2010;19(5):765-77.

24. Ueda H, Kusaba M. Strigolactone regulates leaf senescence in concert with ethylene in Arabidopsis. Plant Physiol. 2015;169(1):138-47.

25. Kim HJ, Ryu H, Hong SH, Woo HR, Lim PO, Lee IC, Sheen J, Nam HG, Hwang I. Cytokinin-mediated control of leaf longevity by AHK3 through phosphorylation of ARR2 in Arabidopsis. Proc Natl Acad Sci U S A. 2006; 103(3):814-9.

26. Chen L, Xiang S, Chen Y, Li D, Yu D. Arabidopsis WRKY45 interacts with the DELLA protein RGL1 to positively regulate age-triggered leaf senescence. Mol Plant. 2017;10(9):1174-89.

27. Kim Jl, Murphy AS, Baek D, Lee SW, Yun DJ, Bressan RA, Narasimhan ML. YUCCA6 over-expression demonstrates auxin function in delaying leaf senescence in Arabidopsis thaliana. J Exp Bot. 2011;62(11):3981-92.

28. Zhang $\mathrm{H}$, Zhou $\mathrm{C}$. Signal transduction in leaf senescence. Plant Mol Biol. 2013;82(6):539-45.

29. Wasternack C. Termination in Jasmonate signaling by MYC2 and MTBs. Trends Plant Sci. 2019;24(8):667-9.

30. Huang H, Liu B, Liu L, Song S. Jasmonate action in plant growth and development. J Exp Bot. 2017;68(6):1349-59.

31. Ueda J, Kato J. Isolation and identification of a senescence-promoting substance from wormwood (Artemisia absinthium L.). Plant Physiol. 1980; 66(2):246-9.

32. He Y, Fukushige $H$, Hildebrand DF, Gan S. Evidence supporting a role of jasmonic acid in Arabidopsis leaf senescence. Plant Physiol. 2002;128(3):876-84.

33. Schommer C, Palatnik JF, Aggarwal P, Chetelat A, Cubas P, Farmer EE, Nath U, Weigel D. Control of jasmonate biosynthesis and senescence by miR319 targets. PLoS Biol. 2008;6(9):e230.

34. Koyama T, Sato F, Ohme-Takagi M. Roles of miR319 and TCP transcription factors in leaf development. Plant Physiol. 2017;175(2):874-85.

35. Seltmann MA, Stingl NE, Lautenschlaeger JK, Krischke M, Mueller MJ, Berger S. Differential impact of lipoxygenase 2 and jasmonates on natural and stress-induced senescence in Arabidopsis. Plant Physiol. 2010;152(4):1940-50.

36. Park JH, Oh SA, Kim YH, Woo HR, Nam HG. Differential expression of senescence-associated mRNAs during leaf senescence induced by different senescence-inducing factors in Arabidopsis. Plant Mol Biol. 1998; 37(3):445-54.

37. Borrill $P$, Harrington SA, Simmonds J, Uauy C. Identification of transcription factors regulating senescence in wheat through gene regulatory network Modelling. Plant Physiol. 2019;180(3):1740-55.

38. Eulgem T, Rushton PJ, Robatzek S, Somssich IE. The WRKY superfamily of plant transcription factors. Trends Plant Sci. 2000;5(5):199-206.

39. Rushton PJ, Somssich IE, Ringler P, Shen QJ. WRKY transcription factors. Trends Plant Sci. 2010;15(5):247-58.
40. Miao Y, Laun T, Zimmermann P, Zentgraf U. Targets of the WRKY53 transcription factor and its role during leaf senescence in Arabidopsis. Plant Mol Biol. 2004;55(6):853-67.

41. Miao Y, Zentgraf U. The antagonist function of Arabidopsis WRKY53 and ESR/ESP in leaf senescence is modulated by the jasmonic and salicylic acid equilibrium. Plant Cell. 2007;19(3):819-30.

42. Ay N, Irmler K, Fischer A, Uhlemann R, Reuter G, Humbeck K. Epigenetic programming via histone methylation at WRKY53 controls leaf senescence in Arabidopsis thaliana. Plant J. 2009;58(2):333-46.

43. Zhang Y, Liu Z, Wang X, Wang J, Fan K, Li Z, Lin W. DELLA proteins negatively regulate dark-induced senescence and chlorophyll degradation in Arabidopsis through interaction with the transcription factor WRKY6. Plant Cell Rep. 2018:37(7):981-92.

44. Robatzek S, Somssich IE. A new member of the Arabidopsis WRKY transcription factor family, AtWRKY6, is associated with both senescenceand defence-related processes. Plant J. 2001;28(2):123-33.

45. Lim J, Park JH, Jung S, Hwang D, Nam HG, Hong S. Antagonistic roles of phy $A$ and phyB in far-red light-dependent leaf senescence in Arabidopsis thaliana. Plant Cell Physiol. 2018;59(9):1753-64.

46. Jiang Y, Liang G, Yang S, Yu D. Arabidopsis WRKY57 functions as a node of convergence for jasmonic acid- and auxin-mediated signaling in jasmonic acid-induced leaf senescence. Plant Cell. 2014;26(1):230-45.

47. Besseau S, Li J, Palva ET. WRKY54 and WRKY70 co-operate as negative regulators of leaf senescence in Arabidopsis thaliana. J Exp Bot. 2012;63(7):2667-79.

48. Lobell DB, Schlenker W, Costa-Roberts J. Climate trends and global crop production since 1980. Science. 2011;333(6042):616-20.

49. Petersen G, Seberg O, Yde M, Berthelsen K. Phylogenetic relationships of Triticum and Aegilops and evidence for the origin of the $a, B$, and D genomes of common wheat (Triticum aestivum). Mol Phylogenet Evol. 2006;39(1):70-82

50. Dubcovsky J, Dvorak J. Genome plasticity a key factor in the success of polyploid wheat under domestication. Science. 2007;316(5833):1862-6.

51. Brenchley R, Spannagl M, Pfeifer M, Barker GL, D'Amore R, Allen AM, McKenzie N, Kramer M, Kerhornou A, Bolser D, et al. Analysis of the bread wheat genome using whole-genome shotgun sequencing. Nature. 2012; 491(7426):705-10.

52. International Wheat Genome Sequencing C. A chromosome-based draft sequence of the hexaploid bread wheat (Triticum aestivum) genome. Science. 2014;345(6194):1251788.

53. Clavijo BJ, Venturini L, Schudoma C, Accinelli GG, Kaithakottil G, Wright J, Borrill P, Kettleborough G, Heavens D, Chapman H, et al. An improved assembly and annotation of the allohexaploid wheat genome identifies complete families of agronomic genes and provides genomic evidence for chromosomal translocations. Genome Res. 2017;27(5):885-96.

54. International Wheat Genome Sequencing $C$, investigators IRp, Appels $R$, Eversole K, Feuillet C, Keller B, Rogers J, Stein N, investigators Iw-gap, Pozniak CJ, et al. Shifting the limits in wheat research and breeding using a fully annotated reference genome. Science. 2018;361(6403):eaar7191.

55. Guo Y, Gan S. AtNAP, a NAC family transcription factor, has an important role in leaf senescence. Plant J. 2006;46(4):601-12.

56. Jaemsaeng $\mathrm{R}$, Jantasuriyarat $\mathrm{C}$, Thamchaipenet $\mathrm{A}$. Molecular interaction of 1 aminocyclopropane-1-carboxylate deaminase (ACCD)-producing endophytic Streptomyces sp. GMKU 336 towards salt-stress resistance of Oryza sativa L. cv. KDML105. Sci Rep. 2018;8(1):1950.

57. Hirel B, Bertin P, Quillere I, Bourdoncle W, Attagnant C, Dellay C, Gouy A Cadiou S, Retailliau C, Falque M, et al. Towards a better understanding of the genetic and physiological basis for nitrogen use efficiency in maize. Plant Physiol. 2001;125(3):1258-70.

58. Barneix AJ. Physiology and biochemistry of source-regulated protein accumulation in the wheat grain. J Plant Physiol. 2007;164(5):581-90.

59. Donnison IS, Gay AP, Thomas H, Edwards KJ, Edwards D, James CL, Thomas AM, Ougham HJ. Modification of nitrogen remobilization, grain fill and leaf senescence in maize (Zea mays) by transposon insertional mutagenesis in a protease gene. New Phytol. 2007:173(3):481-94.

60. Viana VE, Busanello C, da Maia LC, Pegoraro C, Costa de Oliveira A. Activation of rice WRKY transcription factors: an army of stress fighting soldiers? Curr Opin Plant Biol. 2018;45(Pt B):268-75.

61. Hu Z, Wang R, Zheng M, Liu X, Meng F, Wu H, Yao Y, Xin M, Peng H, Ni Z et al. TaWRKY51 promotes lateral root formation through negative regulation of ethylene biosynthesis in wheat (Triticum aestivum L.). Plant J. 2018;96(2):372-88 
62. Gao H, Wang Y, Xu P, Zhang Z. Overexpression of a WRKY transcription factor TaWRKY2 enhances drought stress tolerance in transgenic wheat. Front Plant Sci. 2018;9:997.

63. Niu CF, Wei W, Zhou QY, Tian AG, Hao YJ, Zhang WK, Ma B, Lin Q, Zhang ZB, Zhang JS, et al. Wheat WRKY genes TaWRKY2 and TaWRKY19 regulate abiotic stress tolerance in transgenic Arabidopsis plants. Plant Cell Environ. 2012;35(6):1156-70.

64. Kage U, Yogendra KN, Kushalappa AC. TaWRKY70 transcription factor in wheat QTL-2DL regulates downstream metabolite biosynthetic genes to resist Fusarium graminearum infection spread within spike. Sci Rep. 2017;7 42596.

65. Wu A, Allu AD, Garapati P, Siddiqui H, Dortay H, Zanor Ml, Asensi-Fabado MA, Munne-Bosch S, Antonio C, Tohge T, et al. JUNGBRUNNEN1, a reactive oxygen species-responsive NAC transcription factor, regulates longevity in Arabidopsis. Plant Cell. 2012:24(2):482-506

66. Ju L, Jing Y, Shi P, Liu J, Chen J, Yan J, Chu J, Chen KM, Sun J. JAZ proteins modulate seed germination through interaction with ABI5 in bread wheat and Arabidopsis. New Phytol. 2019;223(1):246-60.

67. Jing Y, Liu J, Liu P, Ming D, Sun J. Overexpression of TaJAZ1 increases powdery mildew resistance through promoting reactive oxygen species accumulation in bread wheat. Sci Rep. 2019;9(1):5691.

68. Zhao Y, Dong W, Zhang N, Ai X, Wang M, Huang Z, Xiao L, Xia G. A wheat allene oxide cyclase gene enhances salinity tolerance via jasmonate signaling. Plant Physiol. 2014;164(2):1068-76.

69. Wang Y, Yuan G, Yuan S, Duan W, Wang P, Bai J, Zhang F, Gao S, Zhang L, Zhao C. TaOPR2 encodes a 12-oxo-phytodienoic acid reductase involved in the biosynthesis of jasmonic acid in wheat (Triticum aestivum L.). Biochem Biophys Res Commun. 2016;470(1):233-8.

70. Chen S, Zhou Y, Chen Y, Gu J. fastp: an ultra-fast all-in-one FASTQ preprocessor. Bioinformatics. 2018;34(17):i884-90.

71. Anders S, Pyl PT, Huber W. HTSeq--a Python framework to work with highthroughput sequencing data. Bioinformatics. 2015;31(2):166-9.

72. Kim D, Langmead B, Salzberg SL. HISAT: a fast spliced aligner with low memory requirements. Nat Methods. 2015;12(4):357-60.

73. Kumar S, Stecher G, Li M, Knyaz C, Tamura K. MEGA X: molecular evolutionary genetics analysis across computing platforms. Mol Biol Evol. 2018;35(6):1547-9.

74. Chen C, Chen H, Zhang Y, Thomas HR, Frank MH, He Y, Xia R. TBtools: An Integrative Toolkit Developed for Interactive Analyses of Big Biological Data. Mol Plant. 2020;13(8):1194-202.

75. Luo Z, Kong X, Zhang Y, Li W, Zhang D, Dai J, Fang S, Chu J, Dong H. Leafderived Jasmonate mediates water uptake from hydrated cotton roots under partial root-zone irrigation. Plant Physiol. 2019;180(3):1660-76

\section{Publisher's Note}

Springer Nature remains neutral with regard to jurisdictional claims in published maps and institutional affiliations.

Ready to submit your research? Choose BMC and benefit from:

- fast, convenient online submission

- thorough peer review by experienced researchers in your field

- rapid publication on acceptance

- support for research data, including large and complex data types

- gold Open Access which fosters wider collaboration and increased citations

- maximum visibility for your research: over $100 \mathrm{M}$ website views per year

At $\mathrm{BMC}$, research is always in progress.

Learn more biomedcentral.com/submissions 